\title{
ON THE EXISTENCE OF SPATIALLY TEMPERED NULL SOLUTIONS TO LINEAR CONSTANT COEFFICIENT PDES
}

\author{
AMOL SASANE
}

\begin{abstract}
Given a linear, constant coefficient partial differential equation in $\mathbb{R}^{d+1}$, where one independent variable plays the role of 'time', a distributional solution is called a null solution if its past is zero. Motivated by physical considerations, we consider distributional solutions that are tempered in the spatial directions alone (and do not impose any restriction in the time direction). Considering such spatially tempered distributional solutions, we give an algebraic-geometric characterization, in terms of the polynomial describing the PDE at hand, for the null solution space to be trivial (that is, consisting only of the zero distribution).
\end{abstract}

\section{INTRODUCTION}

Given a polynomial $p \in \mathbb{C}\left[X_{1}, \cdots, X_{d}, T\right]=: \mathbb{C}[\mathbf{X}, T]$, we associate with it the linear constant coefficient differential operator $D_{p}$ by making the replacements

$$
\begin{aligned}
X_{k} & \rightsquigarrow \frac{\partial}{\partial x_{k}}, \quad k=1, \cdots, d \\
T & \rightsquigarrow \frac{\partial}{\partial t} .
\end{aligned}
$$

Definition 1.1 (Solution space).

A solution space is a subspace $S$ of the space of distributions $\mathcal{D}^{\prime}\left(\mathbb{R}^{d+1}\right)$.

Throughout this article, unless otherwise indicated, we will use the standard distribution theory notation from Schwartz [11] or Tréves [12]. If we fix a solution space $S$, a polynomial $p \in \mathbb{C}[\mathbf{X}, T]$ gives rise to the differential operator $D_{p}: S \rightarrow \mathcal{D}^{\prime}\left(\mathbb{R}^{d+1}\right)$, defined by

$$
D_{p} u:=p\left(\frac{\partial}{\partial x_{1}}, \cdots, \frac{\partial}{\partial x_{d}}, \frac{\partial}{\partial t}\right) u, \quad u \in S
$$

2010 Mathematics Subject Classification. Primary 35A02; Secondary 58J40, 35A24, 35A22, 46F $12,32 \mathrm{C} 25$.

Key words and phrases. partial differential equations, null solutions, distributions that are tempered in the spatial directions. 
Definition 1.2 (Null solution, null solution space). Let $p \in \mathbb{C}[\mathbf{X}, T]$, and $S$ be a solution space. A null solution in $S$ associated with $p$ is a distribution $u \in S$ such that

- (Solution) $D_{p} u=\mathbf{0}$

- (Null past) $\left.u\right|_{t<0}=\mathbf{0}$

We denote by $N_{S}(p)$ the subspace of $S$ consisting of all null solutions in $S$ associated with $p$ :

$$
N_{S}(p):=\left\{u \in S: D_{p} u=\mathbf{0} \text { and }\left.u\right|_{t<0}=\mathbf{0}\right\} .
$$

The notion of a null solution was considered in 4 and [5].

We are interested in giving an algebraic-geometric characterisation of the polynomials $p$ for which $N_{S}(p)$ is just the trivial subspace $\{\mathbf{0}\}$, consisting of only the zero distribution $\mathbf{0}$. Such a characterization is expected to depend on the solution space $S$, as illustrated by the two results below, Propositions 1.3 and 1.4. In the following, $\mathcal{D}\left(\mathbb{R}^{d+1}\right)$ denotes the space of all compactly supported smooth $\left(\mathrm{C}^{\infty}\right)$ functions on $\mathbb{R}^{d+1}$, and $\mathcal{E}^{\prime}\left(\mathbb{R}^{d+1}\right)$ denotes the space of all compactly supported distributions on $\mathbb{R}^{d+1}$.

Proposition 1.3. Let $S=\mathcal{E}^{\prime}\left(\mathbb{R}^{d+1}\right)$ or $S=\mathcal{D}\left(\mathbb{R}^{d+1}\right)$. Let $p \in \mathbb{C}[\mathbf{X}, T]$. Then $N_{S}(p)=\{\mathbf{0}\}$ if and only if $p \neq \mathbf{0}$.

Proof. ('If' part): Suppose that $D_{p} u=\mathbf{0}$ and that $p \neq \mathbf{0}$. By the PayleyWiener-Schwartz theorem [12, Prop. 29.1,p. 307], the Fourier transform $\mathcal{F} u$ of $u$ (with respect to all the variables) can be extended to an entire function on $\mathbb{C}^{d+1}$. Thus $D_{p} u=\mathbf{0}$ yields that for all $\mathbf{z} \in \mathbb{C}^{d+1}$,

$$
p(i \mathbf{z}) \cdot(\mathcal{F} u)(\mathbf{z})=0 .
$$

But the ring $\mathrm{A}\left(\mathbb{C}^{d+1}\right)$ of entire functions in $d+1$ complex variables forms an integral domain. As $p(i \cdot) \neq \mathbf{0}$ in $\mathrm{A}\left(\mathbb{C}^{d+1}\right)$, we conclude that $\mathcal{F} u=\mathbf{0}$, and so $u=\mathbf{0}$ too. Hence $N_{S}(p)=\{\mathbf{0}\}$.

('Only if' part): Suppose that $p=\mathbf{0}$. Then clearly $N_{S}(p)=S \neq\{\mathbf{0}\}$.

On the other hand, when $S=\mathrm{C}^{\infty}\left(\mathbb{R}^{d+1}\right)$ or $\mathcal{D}^{\prime}\left(\mathbb{R}^{d+1}\right)$, using two results due to Hörmander [7, Theorems 8.6.7, 8.6.8], one can show the result below. Here, $\operatorname{deg}(\cdot)$ is used to denote the total degree, which is the maximum (over the monomials $X_{1}^{i_{1}} \cdots X_{d}^{i_{d}} T^{i_{d+1}}$ occurring in the polynomial) of the sum of the degrees of the exponents of indeterminates in the monomial. Also if we decompose $p=p_{m}+p_{m-1}+\cdots+p_{0}$, where $p_{j}$ is homogeneous of total degree $j$ and $p_{m} \neq \mathbf{0}$, then $p_{m}$ is called the principal part of $p$. Let $\langle\cdot, \cdot\rangle_{\mathbb{R}^{d+1}}$ denote the Euclidean inner product on $\mathbb{R}^{d+1}$. Given a vector $\mathbf{n} \in \mathbb{R}^{d+1}$, the plane $\left\{\mathbf{y}:\langle\mathbf{y}, \mathbf{n}\rangle_{\mathbb{R}^{d+1}}=0\right\}$ is called characteristic with respect to $D_{p}$ if $p_{m}(\mathbf{n})=0$.

Proposition 1.4. Let $S=\mathcal{D}^{\prime}\left(\mathbb{R}^{d+1}\right)$ or $\mathrm{C}^{\infty}\left(\mathbb{R}^{d+1}\right)$ and let $p \in \mathbb{C}[\mathbf{X}, T]$. Then $N_{S}(p)=\{\mathbf{0}\}$ if and only if $\operatorname{deg}(p(\mathbf{X}, T))=\operatorname{deg}(p(\mathbf{0}, T))$. 
Proof. We recall that [7, Theorem 8.6.7] says that for a characteristic plane with normal $\mathbf{n}$, there exists a solution in $\mathrm{C}^{\infty}$ whose support is exactly the half-space $\left\{\mathbf{y}:\langle\mathbf{y}, \mathbf{n}\rangle_{\mathbb{R}^{d+1}} \leqslant 0\right\}$. It can be seen that the hyperplane with the normal vector $\mathbf{n}:=(\mathbf{0}, 1) \in \mathbb{R}^{d+1}$ is characteristic with respect to $D_{p}$ if and only if $\operatorname{deg}(p) \neq \operatorname{deg}(p(\mathbf{0}, T))$. This immediately gives the 'only if' part of the proposition.

For the 'if' part, we use [7. Theorem 8.6.8], which says that if $X_{1}, X_{2}$ are open convex sets such that $X_{1} \subset X_{2}$, then the following are equivalent:

- If $u \in \mathcal{D}^{\prime}\left(X_{2}\right)$ satisfies $D_{p} u=\mathbf{0}$ in $X_{2}$ and $\left.u\right|_{X_{1}}=\mathbf{0}$, then $u=\mathbf{0}$ in $X_{2}$.

- Every characteristic hyperplane which intersects $X_{2}$ also intersects $X_{1}$.

Taking $X_{1}=\left\{(\mathbf{x}, t):\langle(\mathbf{x}, t), \mathbf{n}\rangle_{\mathbb{R}^{d+1}}=t<0\right\}$, where $\mathbf{n}:=(\mathbf{0}, 1) \in \mathbb{R}^{d+1}$, and with $X_{2}:=\mathbb{R}^{d+1}$, the above yields the 'if' part of the proposition.

Let us see what happens when we apply Proposition 1.4 in the case of the diffusion equation.

Example 1.5 (Diffusion equation). Consider the diffusion equation

$$
\left(\frac{\partial}{\partial t}-\Delta\right) u=\mathbf{0}
$$

that is, $D_{p} u=\mathbf{0}$, where $p(\mathbf{X}, T)=T-\left(X_{1}^{2}+\cdots+X_{d}^{2}\right)$.

Since $\operatorname{deg}(p(\mathbf{X}, T))=2$, whereas $\operatorname{deg}(p(\mathbf{0}, T))=\operatorname{deg}(T)=1$, Proposition 1.4 implies that $N_{\mathcal{D}^{\prime}\left(\mathbb{R}^{d+1}\right)}(p) \neq\{\mathbf{0}\}$ and $N_{\mathrm{C}^{\infty}\left(\mathbb{R}^{d+1}\right)}(p) \neq\{\mathbf{0}\}$.

In the above example, the outcome is physically unexpected. Indeed, matter diffusion can be modelled by the PDE above, where $u$ is the density of matter. Then zero density up to time $t=0$ should mean that the density stays zero in the future as well. However, the above example shows that one can have 'pathological' null solutions in $\mathrm{C}^{\infty}$ or in $\mathcal{D}^{\prime}$ that are nonzero in the future. On the other hand, if we choose a different, physically motivated solution space in this context, namely functions which at each time instant have a spatial profile belonging to $\mathrm{L}^{1}\left(\mathbb{R}^{d}\right)$, then the associated null solution space is trivial, as expected. The reason that the null solution space is nontrivial in the above example when $S=\mathrm{C}^{\infty}\left(\mathbb{R}^{d+1}\right)$ or $\mathcal{D}^{\prime}\left(\mathbb{R}^{d+1}\right)$ is that there is no growth restriction on the spatial profiles of the solutions at each time instant, and 'rapid' growth 1 is allowed. Indeed, in most physical situations, we expect that at each time instant, the spatial profile is typically in some $\mathrm{L}^{p}$ space or at most polynomially growing, etc. This motivates the following solution space considered in this article. Below $\mathcal{S}\left(\mathbb{R}^{d}\right)$ denotes the Schwartz space of test functions, and $\mathcal{S}^{\prime}\left(\mathbb{R}^{d}\right)$ denotes the space of tempered distributions; see e.g. [12, Chap. 25].

\footnotetext{
${ }^{1}$ Roughly speaking, faster than $e^{\|\mathbf{x}\|^{2}} ;$ see 3 , Theorem, p.44]
} 
Definition 1.6 (Distributions tempered in the spatial directions). The space of distributions on $\mathbb{R}^{d+1}$ tempered in the spatial directions, is the space $\mathcal{L}\left(\mathcal{D}(\mathbb{R}), \mathcal{S}^{\prime}\left(\mathbb{R}^{d}\right)\right.$ ) of all continuous linear maps from $\mathcal{D}(\mathbb{R})$ to $\mathcal{S}^{\prime}\left(\mathbb{R}^{d}\right)$, where $\mathcal{D}(\mathbb{R})$ is endowed with its inductive limit topology and $\mathcal{S}^{\prime}\left(\mathbb{R}^{d}\right)$ is equipped with the weak dual topology $\sigma\left(\mathcal{S}^{\prime}, \mathcal{S}\right)$. We endow $\mathcal{L}\left(\mathcal{D}(\mathbb{R}), \mathcal{S}^{\prime}\left(\mathbb{R}^{d}\right)\right)$ with the topology $\mathcal{L}_{\sigma}\left(\mathcal{D}(\mathbb{R}), \mathcal{S}^{\prime}\left(\mathbb{R}^{d}\right)\right)$ of pointwise convergence.

For $u \in \mathcal{L}\left(\mathcal{D}(\mathbb{R}), \mathcal{S}^{\prime}\left(\mathbb{R}^{d}\right)\right), k=1, \cdots, d$, we define $\frac{\partial u}{\partial x_{k}} \in \mathcal{L}\left(\mathcal{D}(\mathbb{R}), \mathcal{S}^{\prime}\left(\mathbb{R}^{d}\right)\right)$ by

$$
\left\langle\frac{\partial u}{\partial x_{k}}(\varphi), \psi\right\rangle:=-\left\langle u(\varphi), \frac{\partial \psi}{\partial x_{k}}\right\rangle \quad\left(\varphi \in \mathcal{D}(\mathbb{R}), \psi \in \mathcal{S}\left(\mathbb{R}^{d}\right)\right)
$$

For $u \in \mathcal{L}\left(\mathcal{D}(\mathbb{R}), \mathcal{S}^{\prime}\left(\mathbb{R}^{d}\right)\right)$, we define $\frac{\partial u}{\partial t} \in \mathcal{L}\left(\mathcal{D}(\mathbb{R}), \mathcal{S}^{\prime}\left(\mathbb{R}^{d}\right)\right)$ by

$$
\left\langle\frac{\partial u}{\partial t}(\varphi), \psi\right\rangle:=-\left\langle u\left(\varphi^{\prime}\right), \psi\right\rangle \quad\left(\varphi \in \mathcal{D}(\mathbb{R}), \psi \in \mathcal{S}\left(\mathbb{R}^{d}\right)\right) .
$$

Also, for an element $u \in \mathcal{L}\left(\mathcal{D}(\mathbb{R}), \mathcal{S}^{\prime}\left(\mathbb{R}^{d}\right)\right)$, we define its 'spatial' Fourier transform by $\widehat{u} \in \mathcal{L}\left(\mathcal{D}(\mathbb{R}), \mathcal{S}^{\prime}\left(\mathbb{R}^{d}\right)\right)$ by $\langle\widehat{u}(\varphi), \psi\rangle=\langle u(\varphi), \widehat{\psi}\rangle$ for all $\varphi \in \mathcal{D}(\mathbb{R})$ and $\psi \in \mathcal{S}\left(\mathbb{R}^{d}\right)$. Here, for $\psi \in \mathcal{S}^{\prime}\left(\mathbb{R}^{d}\right)$, we define its Fourier transform $\widehat{\psi} \in \mathcal{S}^{\prime}\left(\mathbb{R}^{d}\right)$ by

$$
\widehat{\psi}(\boldsymbol{\xi}):=\int_{\mathbb{R}^{d}} \psi(\mathbf{x}) e^{-i\langle\boldsymbol{\xi}, \mathbf{x}\rangle} \mathbb{R}^{d} \mathrm{~d}^{d} \mathbf{x} \quad\left(\boldsymbol{\xi} \in \mathbb{R}^{d}\right)
$$

where $\langle\cdot, \cdot\rangle_{\mathbb{R}^{d}}$ is the Euclidean inner product on $\mathbb{R}^{d}$.

$\mathcal{L}\left(\mathcal{D}(\mathbb{R}), \mathcal{S}^{\prime}\left(\mathbb{R}^{d}\right)\right)$ can be considered to be a subspace of $\mathcal{D}^{\prime}\left(\mathbb{R}^{d+1}\right)$ as follows. For an element $u \in \mathcal{L}\left(\mathcal{D}(\mathbb{R}), \mathcal{S}^{\prime}\left(\mathbb{R}^{d}\right)\right)$, define $U$ by

$$
\langle U, \varphi \otimes \psi\rangle=\langle u(\varphi), \psi\rangle \quad\left(\varphi \in \mathcal{D}(\mathbb{R}), \psi \in \mathcal{D}\left(\mathbb{R}^{d}\right) \subset \mathcal{S}\left(\mathbb{R}^{d}\right)\right) .
$$

By the Schwartz kernel theorem [7, Theorem 5.2.1, p.128], it follows that there is a unique distribution $U \in \mathcal{D}^{\prime}\left(\mathbb{R}^{d+1}\right)$ such that (11) is satisfied. The space $\mathcal{L}\left(\mathcal{D}(\mathbb{R}), \mathcal{S}^{\prime}\left(\mathbb{R}^{d}\right)\right)$ is also isomorphic to the completed projective- (or 2 epsilon-)tensor product $\mathcal{D}^{\prime}(\mathbb{R}) \widehat{\otimes}_{\pi} \mathcal{S}^{\prime}\left(\mathbb{R}^{d}\right)$ of the spaces $\mathcal{D}^{\prime}(\mathbb{R})$ and $\mathcal{S}^{\prime}\left(\mathbb{R}^{d}\right)$.

We will study the set of null solutions with respect to the space of distributions tempered in the spatial directions, and give an algebraic-geometric characterization of those polynomials $p$ for which the corresponding null solution space consists of just the zero solution. Before we state our result, we give a few algebraic-geometric definitions, and some motivation for arriving at this condition.

\footnotetext{
${ }^{2}$ The projective tensor product topology and the epsilon tensor product topology coincide here, since at least one of the two spaces, and in fact in our case, both $\mathcal{D}^{\prime}(\mathbb{R})$ and $\mathcal{S}^{\prime}\left(\mathbb{R}^{d}\right)$, are nuclear.
} 
Definition 1.7 (Variety).

Given a set $I$ of polynomials from $\mathbb{C}\left[X_{1}, \cdots, X_{d}\right]$, the variety $V(I)$ of $I$ in $\mathbb{C}^{d}$, is the set of all common zeros of the polynomials from $I$, that is, $V(I)=\left\{\boldsymbol{\xi} \in \mathbb{C}^{d}: p(\boldsymbol{\xi})=0\right.$ for all $\left.p \in I\right\}$.

We make the following two observations, leading us to our main result.

(1) Let $p \in \mathbb{C}[\mathbf{X}]$, and let $u \in \mathcal{S}^{\prime}\left(\mathbb{R}^{d}\right)$ be such that $D_{p} u=\mathbf{0}$. Taking Fourier transform, $p(i \boldsymbol{\xi}) \widehat{u}=\mathbf{0}$. So $\operatorname{supp}(\widehat{u}) \subset\left\{\boldsymbol{\xi} \in \mathbb{R}^{d}: p(i \boldsymbol{\xi})=0\right\}$. Thus if $V(p) \cap i \mathbb{R}^{d}=\varnothing$, then $\widehat{u}=\mathbf{0}$, and hence also $u=\mathbf{0}$.

(2) Let $p \in \mathbb{C}[T]$, and let $u \in \mathcal{D}^{\prime}(\mathbb{R})$ be such that $D_{p} u=\mathbf{0}$. Then $u$ is a classical solution, expressible as a linear combination of the real analytic functions $t^{k} e^{\lambda t}$ for some nonnegative integers $k$ and complex numbers $\lambda$. If the past of $u$ is zero, that is, $\left.u\right|_{t<0}=\mathbf{0}$, then $u=\mathbf{0}$.

As our solution space $\mathcal{L}\left(\mathcal{D}(\mathbb{R}), \mathcal{S}^{\prime}\left(\mathbb{R}^{d}\right)\right) \simeq \mathcal{D}^{\prime}(\mathbb{R}) \widehat{\otimes}_{\pi} \mathcal{S}^{\prime}\left(\mathbb{R}^{d}\right)$, we expect our algebraic-geometric characterisation to reduce to above extreme cases when the polynomial belongs either to $\mathbb{C}[\mathbf{X}]$ or to $\mathbb{C}[T]$. In order to formulate this algebraic-geometric condition, we give the following definition.

Definition 1.8 (X-content).

Let $p \in \mathbb{C}[\mathbf{X}, T]$. Writing $p=a_{0}+a_{1} T+\cdots+a_{n} T^{n} \in \mathbb{C}[\mathbf{X}][T]$, where $a_{0}, \cdots, a_{n} \in \mathbb{C}[\mathbf{X}]$, the $\mathbf{X}$-content $C_{\mathbf{X}}(p)$ of $p$ is the ideal in $\mathbb{C}[\mathbf{X}]$ generated by $a_{0}, \cdots, a_{n}$.

We show below that if the variety $V\left(C_{\mathbf{X}}(p)\right)$ of the $\mathbf{X}$-content of $p$ meets $i \mathbb{R}^{d}$, then the null solution space in $\mathcal{L}\left(\mathcal{D}(\mathbb{R}), \mathcal{S}^{\prime}\left(\mathbb{R}^{d}\right)\right)$ associated with $p$ is nontrivial.

\section{Theorem 1.9.}

Let $p \in \mathbb{C}[\mathbf{X}, T]$. If $N_{\mathcal{L}\left(\mathcal{D}(\mathbb{R}), \mathcal{S}^{\prime}\left(\mathbb{R}^{d}\right)\right)}(p)=\{\mathbf{0}\}$, then $V\left(C_{\mathbf{X}}(p)\right) \cap i \mathbb{R}^{d}=\varnothing$.

Proof. Let $V\left(C_{\mathbf{X}}(p)\right) \cap i \mathbb{R}^{d} \neq \varnothing$, and $\boldsymbol{\xi}_{0} \in \mathbb{R}^{d}$ be such that $i \boldsymbol{\xi}_{0} \in V\left(C_{\mathbf{X}}(p)\right)$.

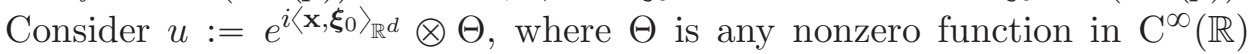
which has a zero past, for example,

$$
\Theta(t)= \begin{cases}0 & \text { if } t \leqslant 0 \\ e^{-1 / t} & \text { if } t>0 .\end{cases}
$$

Then $u \in \mathcal{L}\left(\mathcal{D}(\mathbb{R}), \mathcal{S}^{\prime}\left(\mathbb{R}^{d}\right)\right)$ and it has zero past, that is, $\left.u\right|_{t<0}=\mathbf{0}$. If $p=a_{0}+a_{1} T+\cdots+a_{n} T^{n}$, where $a_{0}, \cdots, a_{n} \in \mathbb{C}[\mathbf{X}]$, then $a_{0}, \cdots, a_{n} \in C_{\mathbf{X}}(p)$, and so $a_{0}\left(i \boldsymbol{\xi}_{0}\right)=\cdots=a_{n}\left(i \boldsymbol{\xi}_{0}\right)=0$.

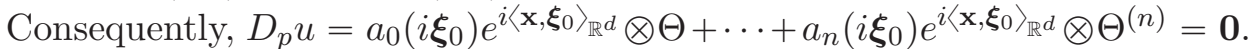
Hence $u \in N_{\mathcal{L}\left(\mathcal{D}(\mathbb{R}), \mathcal{S}^{\prime}\left(\mathbb{R}^{d}\right)\right)}(p)$. But $u \neq \mathbf{0}$, and so $N_{\mathcal{L}\left(\mathcal{D}(\mathbb{R}), \mathcal{S}^{\prime}(\mathbb{R})\right)}(p) \neq\{\mathbf{0}\}$.

In light of the necessity of $V\left(C_{\mathbf{X}}(p)\right) \cap i \mathbb{R}^{d}=\varnothing$ for $N_{\mathcal{L}\left(\mathcal{D}(\mathbb{R}), \mathcal{S}^{\prime}\left(\mathbb{R}^{d}\right)\right)}(p)=\{\mathbf{0}\}$, a natural question is whether this condition is also sufficient. Our main result (Theorem 4.1) is to show the sufficiency. Thus, Theorems 1.9 and 4.1 together give: 
Theorem 1.10.

Let $p \in \mathbb{C}[\mathbf{X}, T] . N_{\mathcal{L}\left(\mathcal{D}(\mathbb{R}), \mathcal{S}^{\prime}\left(\mathbb{R}^{d}\right)\right)}(p)=\{\mathbf{0}\}$ if and only if $V(C \mathbf{X}(p)) \cap i \mathbb{R}^{d}=\varnothing$.

In the last section, we will also consider distributions which have spatial profiles at each time instant lying in certain Besov spaces.

We summarise the results in a table below:

\begin{tabular}{|c|c|c|c|}
\hline & Solution space $S$ & Test on $p$ for $N_{S}(p)=\{\mathbf{0}\}$ & Result reference \\
\hline \hline$(1)$ & $\mathrm{C}^{\infty}\left(\mathbb{R}^{d+1}\right)$ & $\operatorname{deg}(p)=\operatorname{deg}(p(\mathbf{0}, T))$ & Proposition [1.4 \\
\hline$(2)$ & $\mathcal{D}^{\prime}\left(\mathbb{R}^{d+1}\right)$ & $\operatorname{deg}(p)=\operatorname{deg}(p(\mathbf{0}, T))$ & Proposition [1.4 \\
\hline$(3)$ & $\mathcal{D}\left(\mathbb{R}^{d+1}\right)$ & $p \neq \mathbf{0}$ & Proposition [1.3 \\
\hline$(4)$ & $\mathcal{E}^{\prime}\left(\mathbb{R}^{d+1}\right)$ & $p \neq \mathbf{0}$ & Proposition [1.3 \\
\hline$(5)$ & $\mathcal{L}\left(\mathcal{D}(\mathbb{R}), \mathcal{S}^{\prime}\left(\mathbb{R}^{d}\right)\right)$ & $V\left(C_{\mathbf{X}}(p)\right) \cap i \mathbb{R}^{d}=\varnothing$ & Theorems [1.9, [4.1 \\
\hline$(6)$ & $\mathcal{L}\left(\mathcal{D}(\mathbb{R}), B_{p, q}\left(\mathbb{R}^{d}\right)\right)$ & $p \neq \mathbf{0}$ & Theorem [5.1] \\
\hline$(7)$ & $\mathcal{L}\left(\mathcal{D}(\mathbb{R}), H_{s}\left(\mathbb{R}^{d}\right)\right)$ & $p \neq \mathbf{0}$ & Corollary [5.2 \\
\hline$(8)$ & $\mathcal{L}\left(\mathcal{D}(\mathbb{R}), \mathcal{S}\left(\mathbb{R}^{d}\right)\right)$ & $p \neq \mathbf{0}$ & Corollary [5.2 \\
\hline$(9)$ & $\mathcal{L}\left(\mathcal{D}(\mathbb{R}), \mathcal{E}^{\prime}\left(\mathbb{R}^{d}\right)\right)$ & $p \neq \mathbf{0}$ & Theorem [5.3 \\
\hline$(10)$ & $\mathcal{D}_{\mathbb{A}}^{\prime}\left(\mathbb{R}^{d+1}\right)$ & $\forall \mathbf{v} \in A^{-1} 2 \pi \mathbb{Z}^{d}, \exists t \in \mathbb{C}:$ & Theorem 6.1] \\
& & $p(i \mathbf{v}, t) \neq 0$. & \\
\hline
\end{tabular}

The key idea used in proving the sufficiency part is as follows. By taking Fourier transform, the partial derivatives $\partial_{x_{k}}$ with respect to the spatial variables $x_{k}$ are converted into $i \xi_{k}$, and so we obtain $p\left(i \boldsymbol{\xi}, \partial_{t}\right) \widehat{u}=\mathbf{0}$, a family, parameterised by $\boldsymbol{\xi} \in \mathbb{R}^{d}$, of equations involving $\partial_{t}^{k}$ with the polynomial coefficients $a_{k}(i \boldsymbol{\xi})$. One would like to 'freeze' a $\boldsymbol{\xi} \in \mathbb{R}^{d}$, to get an ODE for $(\widehat{u}(\cdot))(\boldsymbol{\xi}) \in \mathcal{D}^{\prime}(\mathbb{R})$, where for such a solution to an ODE we can indeed say that zero past implies zero future, and so the proof can be completed easily by varying the arbitrarily fixed $\boldsymbol{\xi}$. This is possible if the spatial Fourier transform is a function, so that the evaluation at $\boldsymbol{\xi}$ is allowed, and this is essentially how one shows the results (6)-(10).

For showing our main result (5), where spatial Fourier transform will not result in a function of $\boldsymbol{\xi}$, but rather a distribution, the idea is as follows. Using Holmgren's uniqueness principle, the support of $\widehat{u}$ is contained in $V \times[0, \infty)$, where $V$ is the real zero set of the leading coefficient $a_{n}$. If $d=1$, so that $a_{n}$ were a polynomial of just one variable, then the real zeroes are isolated points, and we can complete the proof using a structure theorem of Schwartz, which says that distributions supported on a line must be essentially the Dirac delta and its derivatives, tensored with distributions $T_{k}$ of one variable (time). We can then boil down $p\left(i \boldsymbol{\xi}, \partial_{t}\right) \widehat{u}=\mathbf{0}$ to give an ODE for these distributions $T_{k}$ of time, and since each $T_{k}$ can be shown to have zero past, we can conclude that the $T_{k} \mathrm{~s}$ must be zero. So this is how the proof works when $d=1$ and when $a_{n}$ was a polynomial of just one variable. In the general case, to handle the case when $a_{n}$ may be a polynomial of $d$ 
variables, we proceed inductively on the number of spatial dimensions $d$. It is too much to hope that at each inductive step we end up with polynomials as coefficients of $\partial_{t}^{k}$, since polynomial parametrisations of the zero sets of the polynomial $a_{n}$ may not be possible (e.g. $\left\{(X, Y): X^{2}+Y^{2}-1=0\right\}$ does not possess a polynomial parametrisation). But the $d=1$ case just relied on the discreteness of the zero set of $a_{n}$, which is also guaranteed if $a_{n}$ were real analytic instead of being a polynomial. So to carry out the induction, we use the set up where we make sure that the coefficients of $\partial_{t}^{k}$ obtained at each inductive step are real analytic functions. To begin with, polynomials are real analytic, real analytic varieties do possess locally real analytic parametrisations (Łojaciewicz structure theorem for real analytic varieties), and composition of real analytic functions is real analytic. This allows us to complete the induction step, by again appealing to a structure theorem of Schwartz, now for distributions with support in a smooth manifold. The technical details are carried out in Lemma 3.1.

The organisation of the article is as follows.

- In Section 2, we recall some preliminaries needed for the proofs.

- In Section 3, we will prove the central technical result in Lemma 3.1. which will lead to the proof of Theorem 4.1 on the sufficiency.

- In Section 4, we will prove Theorem 4.1.

- In Section 5, we consider distributions which have spatial profiles at each time instant lying in certain Besov spaces.

- In Section 6 we consider distributions that are periodic in the spatial directions.

- Finally, in the last section, we mention a class of auxiliary open problems on the theme of null solutions.

\section{Preliminaries}

In this section, we recall three auxiliary known results needed for the proof of Lemma 3.1.

- Holmgren's uniqueness theorem,

- Schwartz structure theorem for distributions supported on a manifold,

- Łojaciewicz structure theorem for real analytic varieties.

2.1. Holmgren's uniqueness theorem. Before recalling this result, we first we establish some terminology and notation. Let $\Omega \subset \mathbb{R}^{d}$ be an open set, and consider the differential operator $P$ with real analytic coefficients $a_{\mathbf{n}}$ of order $N$ :

$$
P=P(x, \boldsymbol{\partial})=\sum_{|\mathbf{n}| \leqslant N} a_{n}(\mathbf{x}) \frac{\partial^{n_{1}}}{\partial x_{1}^{n_{1}}} \cdots \frac{\partial^{n_{d}}}{\partial x_{d}^{n_{d}}} .
$$

Here, for a multi-index $\mathbf{n}=\left(n_{1}, \cdots, n_{d}\right)$ of nonnegative integers, we define $|\mathbf{n}|:=n_{1}+\cdots+n_{d}$. We now recall the following version of the uniqueness theorem of Holmgren [6, Lemma 5.3.2, p.125]: 
Proposition 2.1 (Holmgren's uniqueness theorem). In an open subset $\Omega$ of $\mathbb{R}^{d}$, let

$$
P(\mathbf{x}, \boldsymbol{\partial})=\sum_{|\mathbf{n}| \leqslant N} a_{n}(\mathbf{x}) \frac{\partial^{n_{1}}}{\partial x_{1}^{n_{1}}} \cdots \frac{\partial^{n_{d}}}{\partial x_{d}^{n_{d}}}
$$

be a differential operator having coefficients real analytic on $\Omega$.

Assume that the coefficient of $\frac{\partial^{N}}{\partial x_{d}^{N}}$ never vanishes in $\Omega$.

If $u \in \mathcal{D}^{\prime}(\Omega)$ and $P(\mathbf{x}, \boldsymbol{\partial}) u=0$ in $\Omega_{c}:=\left\{\mathbf{x} \in \Omega: x_{d}<c\right\}$ for some $c$, then $u=\mathbf{0}$ in $\Omega_{c}$ provided that $\Omega_{c} \cap(\operatorname{supp}(u))$ is relatively compact in $\Omega$.

We will use the following consequence of this.

\section{Lemma 2.2.}

Let

- $U$ be an open subset of $\mathbb{R}^{d}$,

- $c_{0}, \cdots, c_{N}$ are real analytic functions of $d$ variables in $U$,

- $u \in \mathcal{D}^{\prime}(U \times \mathbb{R})$,

- $\left.u\right|_{t<0}=\mathbf{0}$,

- $c_{0}(\boldsymbol{\xi}) u+c_{1}(\boldsymbol{\xi}) \frac{\partial u}{\partial t}+\cdots+c_{N}(\boldsymbol{\xi}) \frac{\partial^{N} u}{\partial t^{N}}=\mathbf{0}$.

Then $\operatorname{supp}(u) \subset\left\{(\boldsymbol{\xi}, t) \in U \times \mathbb{R}: c_{N}(\boldsymbol{\xi})=0\right\}$.

Proof. Let $\boldsymbol{\xi}_{0} \in U$ be such that $c_{N}\left(\boldsymbol{\xi}_{0}\right) \neq 0$. Let $r>0$ be such that the open ball $B\left(\boldsymbol{\xi}_{0}, 2 r\right) \subset U$ and $c_{N}(\boldsymbol{\xi}) \neq 0$ in $B\left(\boldsymbol{\xi}_{0}, 2 r\right)$. We will use Holmgren's uniqueness theorem with $\Omega_{\xi_{0}}:=B\left(\boldsymbol{\xi}_{0}, 2 r\right) \times \mathbb{R}$ and the differential operator

$$
P((\boldsymbol{\xi}, t), \boldsymbol{\partial}):=c_{0}(\boldsymbol{\xi})+c_{1}(\boldsymbol{\xi}) \frac{\partial}{\partial t}+\cdots+c_{N}(\boldsymbol{\xi}) \frac{\partial^{N}}{\partial t^{N}} .
$$

The coefficient $c_{N}(\boldsymbol{\xi})$ of $\frac{\partial^{N}}{\partial t^{N}}$ never vanishes in $\Omega_{\boldsymbol{\xi}_{0}}$.

Let $\tilde{u}$ be the restriction of $u$ to $\Omega_{\boldsymbol{\xi}_{0}}=B\left(\boldsymbol{\xi}_{0}, 2 r\right) \times \mathbb{R} \subset U \times \mathbb{R}$. We already know that $\operatorname{supp}(\widetilde{u}) \subset B\left(\boldsymbol{\xi}_{0}, 2 r\right) \times[0, \infty)$ since $\left.\widetilde{u}\right|_{t<0}=\mathbf{0}$ (which in turn follows from $\left.\left.u\right|_{t<0}=\mathbf{0}\right)$. For any $c>0$, with $\Omega_{c}:=\left\{(\boldsymbol{\xi}, t): \boldsymbol{\xi} \in B\left(\boldsymbol{\xi}_{0}, r\right)\right.$ and $\left.t<c\right\}$, we have $\Omega_{c} \cap \operatorname{supp}(\widetilde{u})$ is relatively compact in $\Omega_{\xi_{0}}$. Hence $\widetilde{u}=\mathbf{0}$ in $\Omega_{c}$. As the choice of $c>0$ was arbitrary, it follows that $\tilde{u}=\mathbf{0}$ in $B\left(\boldsymbol{\xi}_{0}, r\right) \times \mathbb{R}$. By varying the $\boldsymbol{\xi}_{0}$ having the property that $c_{N}\left(\boldsymbol{\xi}_{0}\right) \neq 0$, we obtain that the restriction of $u$ to the set $V:=\left\{\boldsymbol{\xi} \in U: c_{N}(\boldsymbol{\xi}) \neq 0\right\} \times \mathbb{R}$ is the zero distribution $\mathbf{0} \in \mathcal{D}^{\prime}(V)$. So $\operatorname{supp}(u) \subset\left\{(\boldsymbol{\xi}, t) \in U \times \mathbb{R}: c_{N}(\boldsymbol{\xi})=0\right\}$. 
2.2. Schwartz structure theorem for distributions with support in a submanifold of $\mathbb{R}^{d}$. We will need a local structure result, due to Schwartz [11, Theorem XXXVII, page 102], for distributions with support contained in a smooth submanifold of $\mathbb{R}^{d}$ (analogous to the well-known structure theorem saying that a distribution with support in a point is a linear combination of the Dirac delta distribution at that point and its derivatives). But before stating this result, it is useful to keep the following guiding example in mind.

Consider in $\mathbb{R}^{d}$ the manifold $M=S^{d-1}$, namely the unit $(d-1)$-dimensional sphere. Let $\partial_{r}$ denote the radial partial derivative. Then we expect that a distribution $T$ in $\mathbb{R}^{d}$ having its support in $S^{d-1}$ should be decomposable as

$$
T=\sum_{k=0}^{K} \partial_{r}^{k} T_{k},
$$

for some distributions $T_{k}$ on $S^{d-1}$, where the action of the right-hand side above on a test function $\varphi=\varphi\left(r, \theta_{1}, \cdots, \theta_{d-1}\right)$ (in an appropriate chart) is understood as

$$
\sum_{k=0}^{K}(-1)^{k}\left\langle T_{k},\left.\partial_{r}^{k} \varphi(r, \cdot)\right|_{r=1}\right\rangle .
$$

A generalisation of this is given below; see [11, Theorem XXXVII, p. 102]. Here, for a multi-index $\mathbf{k}=\left(k_{d^{\prime}+1}, \cdots, k_{d}\right)$ of nonnegative integers, we define $|\mathbf{k}|=k_{d^{\prime}+1}+\cdots+k_{d}$, and

$$
\partial_{\mathbf{y}}^{\mathbf{k}}=\left(\frac{\partial}{\partial y_{d^{\prime}+1}}\right)^{k_{d^{\prime}+1}} \cdots\left(\frac{\partial}{\partial y_{d}}\right)^{k_{d}} .
$$

Proposition 2.3 (Schwartz structure theorem). Suppose that

- $M$ is a submanifold of $\mathbb{R}^{d}$ of dimension $d^{\prime}$,

- $\boldsymbol{\xi}_{0} \in M$, and

- $\mathbf{y}$ are coordinates in $B\left(\boldsymbol{\xi}_{0}, R\right)=\left\{\boldsymbol{\xi} \in \mathbb{R}^{d}:\left\|\boldsymbol{\xi}-\boldsymbol{\xi}_{0}\right\|_{2}<R\right\}$ in $\mathbb{R}^{d}$, such that $B\left(\boldsymbol{\xi}_{0}, R\right) \cap M=\left\{\boldsymbol{\xi} \in B\left(\boldsymbol{\xi}_{0}, R\right): y_{d^{\prime}+1}(\boldsymbol{\xi})=\cdots=y_{d}(\boldsymbol{\xi})=0\right\}$.

Then a distribution $T$ on $\mathbb{R}^{d}$ with support in $M$ can be locally decomposed as

$$
T=\sum_{|\mathbf{k}| \leqslant K} \partial_{\mathbf{y}}^{\mathbf{k}} T_{\mathbf{k}},
$$

for some distributions $T_{\mathbf{k}}$ on $M$.

2.3. Łojasiewicz structure theorem for real analytic varieties. We use the terminology and notation from 9 . Let $U \subset \mathbb{R}^{d}$ be open. Then $\mathrm{C}^{\omega}(U)$ denotes the commutative ring (with respect to pointwise addition and multiplication) of all (possibly complex-valued) real analytic functions in $d$ real variables; see [9, Definition 1.1.5, p.3]. In order to prove our main result in the form of the technical result, namely Lemma 3.1, we will need a structure theorem for real analytic varieties given in Lemma 2.5 below. 
Roughly speaking, this result says that the zero set of a real analytic function of $d$-variables admits a decomposition 3 into the union of real analytic manifolds of various dimensions $\leqslant d-1$. Lemma 2.5 is a consequence of a more elaborate structure theorem for real analytic varieties due to S. Eojaciewicz [10] (see also [1]), which we first recall below. We quote this result after recalling a few pertinent definitions.

A function $H\left(x_{1}, \cdots, x_{d-1} ; x_{d}\right)$ of $d$ real variables is called a distinguished polynomial if it has the form

$$
\begin{aligned}
H\left(x_{1}, \cdots, x_{d-1} ; x_{d}\right)= & x_{d}^{m}+A_{1}\left(x_{1}, \cdots, x_{d-1}\right) x_{d}^{m-1}+\cdots \\
& +A_{m-1}\left(x_{1}, \cdots, x_{d-1}\right) x_{d}+A_{m}\left(x_{1}, \cdots, x_{d-1}\right),
\end{aligned}
$$

where each analytic $A_{\ell}$ vanishes at $\left(x_{1}, \cdots, x_{d-1}\right)=\mathbf{0} \in \mathbb{R}^{d-1}$.

Since the polynomial

$$
\prod_{1 \leqslant i<j \leqslant m}\left(X_{i}-X_{j}\right)^{2} \in \mathbb{Z}\left[X_{1}, \cdots, X_{m}\right]
$$

is symmetric, the theorem on symmetric polynomials [10, p.24] implies the existence of a unique polynomial $\Delta_{m} \in \mathbb{Z}\left[Y_{1}, \cdots, Y_{m}\right]$ such that

$$
\prod_{1 \leqslant i<j \leqslant m}\left(X_{i}-X_{j}\right)^{2}=\Delta_{m}\left(\sigma_{1}, \cdots, \sigma_{m}\right),
$$

where

$$
\sigma_{i}=(-1)^{i} \sum_{\nu_{1}<\cdots<\nu_{i}} X_{\nu_{1}} \cdots X_{\nu_{i}}, \quad i=1, \cdots, m
$$

Let $R$ be a commutative unital ring. The discriminant of a monic polynomial $p=X^{m}+a_{m-1} X^{m-1}+\cdots+a_{m} \in R[X]$, is defined to be $\Delta_{m}\left(a_{1}, \cdots, a_{m}\right) \in R$.

We recall [9, Theorem 6.3.3, p.168] below.

Proposition 2.4 (Łojaciewicz structure theorem). Let $f\left(x_{1}, \cdots, x_{d}\right)$ be a real analytic function in a neighbourhood of the origin. We may assume that $f\left(0, \cdots, 0, x_{d}\right) \not \equiv 0$. After a rotation of the coordinates $x_{1}, \cdots, x_{d-1}$, if needed, there exist $\delta_{j}>0, j=1, \cdots, d$, and distinguished polynomials $H_{\ell}^{k}\left(x_{1}, \cdots, x_{k} ; x_{\ell}\right) \quad(0 \leqslant k \leqslant d-1, k+1 \leqslant \ell \leqslant d)$ defined on $Q_{k}:=\left\{\left|x_{j}\right|<\delta_{j}, 1 \leqslant j \leqslant k\right\}$ such that the discriminant $\Delta_{\ell}^{k}$ of $H_{\ell}^{k}$ does not vanish on $Q_{k}$ and the following properties are satisfied:

(1) Each root $\zeta$ of $H_{\ell}^{k}\left(x_{1}, \cdots, x_{k} ; \cdot\right)$ on $Q_{k}$ satisfies $|\zeta|<\delta_{\ell}$.

(2) The set $V:=\left\{\mathbf{x}=\left(x_{1}, \cdots, x_{d}\right): \forall j\left|x_{j}\right|<\delta_{j}\right.$ and $\left.f(\mathbf{x})=0\right\}$ has a decomposition $V=M_{d-1} \cup \cdots \cup M_{0}$. The set $M_{0}$ is either empty or consists

\footnotetext{
${ }^{3}$ This is analogous to a result due to Whitney [13], saying that a real algebraic variety $V$ can always be decomposed into a union of 'algebraic partial manifolds' of decreasing dimensions: For example, if $V$ is the curve $V=\left\{(x, y) \in \mathbb{R}^{2}: x^{2}-y^{3}=0\right\}$ (with a 'cusp' at the origin), then we have $V=M_{1} \cup M_{2}$, where $M_{1}$ is the curve minus the origin, and $M_{2}=\{(0,0)\}$.
} 
of the origin alone. For $1 \leqslant k \leqslant d-1$, we may write $M_{k}$ as a finite, disjoint union

$$
M_{k}=\bigcup_{\chi} \Gamma_{\chi}^{k}
$$

of $k$-dimensional sub-real analytic varieties having the following description: (Real analytic parametrisation) Each $\Gamma_{\chi}^{k}$ is defined by $d-k$ equations

$$
x_{k+1}=\chi_{\eta_{k+1}^{k}}\left(x_{1}, \cdots, x_{k}\right), \cdots, \quad x_{d}=\chi_{\eta_{d}^{k}}\left(x_{1}, \cdots, x_{k}\right),
$$

where each $\chi_{\eta_{\ell}^{k}}$ is real analytic on an open subset $\Omega_{\chi}^{k} \subset Q_{k} \subset \mathbb{R}^{k}$,

$$
H_{\ell}^{k}\left(x_{1}, \cdots, x_{k} ; \chi_{\eta_{\ell}^{k}}\right) \equiv 0
$$

and $\Delta_{\ell}^{k} \neq 0$ for all $\left(x_{1}, \cdots, x_{k}\right) \in \Omega_{\chi}^{k}, \ell=k+1, \cdots, d$.

(Stratification) For each $k$, the closure of $M_{k}$ contains all the subsequent $M_{j}$ : that is, $Q \cap M_{k} \supset M_{k-1} \cup \cdots \cup M_{0}$.

This result is stronger than what we need. We will only require the decomposition into lower dimensional real analytic varieties and the local analytic parametrisation. We state this as the following corollary of the above.

Lemma 2.5. Let $f: \mathbb{R}^{d} \rightarrow \mathbb{R}$ be a real analytic function, and

$$
V:=\left\{\mathbf{x} \in \mathbb{R}^{d}: f(\mathbf{x})=0\right\}
$$

be its variety. Then for each point $\mathbf{x}_{0}$ of $V$, there exists a neighbourhood $\Omega$ such that there exists a decomposition $V \cap \Omega=M_{d-1} \cup \cdots \cup M_{0}$, where some of the $M_{d^{\prime}} s$ may be empty, and where the $M_{d^{\prime}}$ are a real analytic varieties which are analytic submanifolds of $\mathbb{R}^{d}$ of dimension $d^{\prime}$, admitting real analytic parametrisations as follows: For every $\mathbf{x}_{0} \in M_{d^{\prime}}$, there exists a neighbourhood $U$ of $\mathbf{x}_{0}$ in $\mathbb{R}^{d}$ and a neighbourhood $W$ of $\mathbf{0} \in \mathbb{R}^{d}$, with a homeomorphism $\varphi: W \rightarrow U$ which is real analytic,

$$
\mathbb{R}^{d^{\prime}} \times \mathbb{R}^{d-d^{\prime}} \supset W \ni(\boldsymbol{\tau}, \boldsymbol{\sigma}) \mapsto \varphi(\boldsymbol{\tau}, \boldsymbol{\sigma}) \in U,
$$

such that $M_{d^{\prime}} \cap U=\left\{\varphi(\boldsymbol{\tau}, \mathbf{0}): \boldsymbol{\tau} \in \mathbb{R}^{d^{\prime}}\right.$ such that $\left.(\boldsymbol{\tau}, \mathbf{0}) \in W\right\}$.

Proof. This follows immediately from the above result, since (2) guarantees the local decomposition, and the real analytic parametrisation, namely

$$
\left(x_{1}, \cdots, x_{k}\right) \mapsto\left(x_{1}, \cdots, x_{k}, \chi_{\eta_{k+1}^{k}}\left(x_{1}, \cdots, x_{k}\right), \cdots, \chi_{\eta_{d}^{k}}\left(x_{1}, \cdots, x_{k}\right)\right)
$$

corresponds to the one needed in the statement of the lemma if we take $\boldsymbol{\tau}=\left(x_{1}, \cdots, x_{k}\right), \boldsymbol{\sigma}=\left(x_{k+1}, \cdots, x_{d}\right)$, and

$$
\boldsymbol{\xi}(\boldsymbol{\tau}, \boldsymbol{\sigma})=\left(\boldsymbol{\tau}, \chi_{\eta_{k+1}^{k}}\left(x_{1}, \cdots, x_{k}\right)-x_{k+1}, \cdots, \chi_{\eta_{d}^{k}}\left(x_{1}, \cdots, x_{k}\right)-x_{d}\right) .
$$

Then we note that the differential of $\boldsymbol{\xi}$ has the form

$$
d \boldsymbol{\xi}=\left[\begin{array}{cc}
I_{d^{\prime}} & \mathbf{0} \\
* & -I_{d-d^{\prime}}
\end{array}\right],
$$

which is clearly invertible. Here $I_{k}$ denotes the $k \times k$ identity matrix with ones on the diagonal and zeroes elsewhere. 


\section{The MAIN TECHNiCAL LEMma}

In this section, we will show the main technical result in Lemma 3.1, which will enable us to show our result on sufficiency, namely Theorem 4.1

Lemma 3.1. Let

(1) $U \subset \mathbb{R}^{d}$ be open,

(2) $\mathbf{0} \neq p=c_{0}(\boldsymbol{\xi})+c_{1}(\boldsymbol{\xi}) T+\cdots+c_{n}(\boldsymbol{\xi}) T^{n} \in \mathrm{C}^{\omega}(U)[T], c_{n} \neq \mathbf{0}$,

(3) $V\left(c_{0}, c_{1}, \cdots, c_{n}\right) \cap U=\varnothing$,

(4) $w \in \mathcal{D}^{\prime}(U \times \mathbb{R})$,

(5) $\left.w\right|_{t<0}=\mathbf{0}$,

(6) $c_{0}(\boldsymbol{\xi})+c_{1}(\boldsymbol{\xi}) \frac{\partial}{\partial t} U+\cdots+c_{n}(\boldsymbol{\xi}) \frac{\partial^{n}}{\partial t^{n}} w=\mathbf{0}$.

Then $w=\mathbf{0}$.

Proof. We prove this inductively on the number of spatial dimensions $d$.

Step 1. Let $d=1$. Holmgren's uniqueness theorem (Lemma 2.2) implies

$$
\operatorname{supp}(w) \subset\left\{(\xi, t) \in U \times \mathbb{R}: c_{n}(\xi)=0, t \geqslant 0\right\} .
$$

If $c_{n}$ is constant (which must necessarily be $\neq 0$, since $c_{n}$ was nonzero), then $w=\mathbf{0}$, and we are done.

Let $c_{n}$ be not a constant. Suppose that $w \neq 0$. Let $\left(\xi_{k}\right)_{k \in \mathbb{N}}$ be the real zeros of $c_{n}$ in $U$. Then each $\xi_{k}$ is isolated in $U$. We have that

$$
\operatorname{supp}(w) \subset \bigcup_{k \in \mathbb{N}}\left\{\xi_{k}\right\} \times[0,+\infty)
$$

Each of the half lines above carries a solution of the differential equation

$$
c_{0}(\boldsymbol{\xi})+c_{1}(\boldsymbol{\xi}) \frac{\partial}{\partial t} U+\cdots+c_{n}(\boldsymbol{\xi}) \frac{\partial^{n}}{\partial t^{n}} w=\mathbf{0},
$$

and $w$ is a sum of these.

Let $T \in(0, \infty)$. Take a $\xi_{*} \in\left\{\xi_{1}, \xi_{2}, \cdots\right\}, U$ a neighbourhood of $\xi_{*}$ not containing the other $\xi_{k} \mathrm{~s}$, and an $\alpha \in \mathcal{D}(\mathbb{R})$ which is identically 1 in a neighbourhood of $[-T, T]$ such that the distribution $\alpha w \in \mathcal{D}^{\prime}(U \times \mathbb{R})$ is nonzero. Then $\alpha w$ has compact support, and by the structure theorem for distributions (e.g. [7, Theorem 2.3.5, p.47] or the result from Subsection 2.2), it follows that there exist distributions $T_{0}, \cdots, T_{K} \in \mathcal{D}^{\prime}(\mathbb{R})$ ('of the time variable'), with $T_{K} \neq \mathbf{0}$, such that

$$
\alpha w=\sum_{k=0}^{K}\left(\left(\frac{\partial}{\partial \xi}\right)^{k} \delta_{\xi_{*}}\right) \otimes T_{k} .
$$


Here $\delta_{\xi_{*}}$ is the Dirac delta of the spatial variable $\xi$, supported at $\xi_{*}$. From the above, it can be shown that also

$$
w=\sum_{k=0}^{K}\left(\left(\frac{\partial}{\partial \xi}\right)^{k} \delta_{\xi_{*}}\right) \otimes T_{k}
$$

in the strip $U \times(-T, T)$.

We claim that $\left.T_{K}\right|_{(-T, 0)}=\mathbf{0}$. For if not, then there is a $\varphi \in \mathcal{D}(\mathbb{R})$ with support in $(-T, 0)$ such that $\left\langle T_{K}, \varphi\right\rangle \neq 0$. Hence the sum

$$
\sum_{k=0}^{K}\left\langle T_{k}, \varphi\right\rangle\left(\frac{\partial}{\partial \xi}\right)^{k} \delta_{\xi *}
$$

is a nonzero distribution in $\mathcal{D}^{\prime}(U)$. Otherwise, we get the contradiction that $\delta_{\xi_{*}}, \cdots, \delta_{\xi_{*}}^{(K)}$ are linearly dependent in $\mathcal{D}^{\prime}(U)$. So there must exist a $\psi \in \mathcal{D}(U)$ such that

$$
\left\langle\sum_{k=0}^{K}\left\langle T_{k}, \varphi\right\rangle\left(\frac{\partial}{\partial \xi}\right)^{k} \delta_{\xi_{*}}, \psi\right\rangle \neq 0
$$

that is, $\langle w, \psi \otimes \varphi\rangle \neq 0$. But the support of $\psi \otimes \varphi$ is in $U \times(-T, 0)$, and so we have arrived at a contradiction to $\left.w\right|_{t<0}=\mathbf{0}$. This proves $\left.T_{K}\right|_{(-T, 0)}=\mathbf{0}$.

Using

$$
\mathbf{0}=\sum_{\ell=0}^{n} c_{\ell}(\xi)\left(\frac{\partial}{\partial t}\right)^{\ell} w
$$

we have that for $\left(\xi-\xi_{*}\right)^{K} \in \mathrm{C}^{\infty}(U)$ and $\varphi \in \mathcal{D}(\mathbb{R})$, that

$$
\begin{aligned}
0 & =\left\langle\sum_{\ell=0}^{n} c_{\ell}(\xi)\left(\frac{\partial}{\partial t}\right)^{\ell} \sum_{k=0}^{K}\left(\frac{\partial}{\partial \xi}\right)^{k} \delta_{\xi_{*}} \otimes T_{k},\left(\xi-\xi_{*}\right)^{K} \otimes \varphi\right\rangle \\
& =\sum_{\ell=0}^{n} \sum_{k=0}^{K}\left\langle\left(\frac{\partial}{\partial t}\right)^{\ell} T_{k}, \varphi\right\rangle(-1)^{k}\left\langle\delta_{\xi_{*}},\left(\frac{\partial}{\partial \xi}\right)^{k}\left(c_{\ell}(\xi)\left(\xi-\xi_{*}\right)^{K}\right)\right\rangle .
\end{aligned}
$$

But by the Leibniz product rule,

$$
\left(\frac{\partial}{\partial \xi}\right)^{k}\left(c_{\ell}(\xi)\left(\xi-\xi_{*}\right)^{K}\right)=\sum_{m=0}^{k}\left(\begin{array}{c}
k \\
m
\end{array}\right)\left(\left(\xi-\xi_{*}\right)^{K}\right)^{(m)}\left(\frac{\partial}{\partial \xi}\right)^{k-m} c_{\ell}(\xi),
$$

and if $k<K$, then for all $m=0, \cdots, k$, the $m$ th derivative of $\left(\xi-\xi_{*}\right)^{K}$ will be zero at $\xi=\xi_{*}$ as $K-m \geqslant K-k \geqslant 1$. So for $k<K$,

$$
\left.\left(\frac{\partial}{\partial \xi}\right)^{k}\left(c_{\ell}(\xi)\left(\xi-\xi_{*}\right)^{K}\right)\right|_{\xi=\xi_{*}}=0
$$


Hence the sum over $k=0, \cdots, K$ in (4) collapses to one over $k=K$, giving

$$
\begin{aligned}
0 & =\sum_{\ell=0}^{n}\left\langle\left(\frac{\partial}{\partial t}\right)^{\ell} T_{K}, \varphi\right\rangle(-1)^{K}\left\langle\delta_{\xi_{*}},\left(\frac{\partial}{\partial \xi}\right)^{K}\left(c_{\ell}(\xi)\left(\xi-\xi_{*}\right)^{K}\right)\right\rangle \\
& =\sum_{\ell=0}^{n}\left\langle\left(\frac{\partial}{\partial t}\right)^{\ell} T_{K}, \varphi\right\rangle(-1)^{K} c_{\ell}\left(\xi_{*}\right) K ! \\
& =(-1)^{K} K !\left\langle\sum_{\ell=0}^{n} c_{\ell}\left(\xi_{*}\right)\left(\frac{\partial}{\partial t}\right)^{\ell} T_{K}, \varphi\right\rangle .
\end{aligned}
$$

As the choice of $\varphi \in \mathcal{D}(\mathbb{R})$ was arbitrary, it follows that

$$
\left(c_{0}\left(\xi_{*}\right)+c_{1}\left(\xi_{*}\right) \frac{d}{d t}+\cdots+c_{n}\left(\xi_{*}\right)\left(\frac{d}{d t}\right)^{n}\right) T_{K}=\mathbf{0} .
$$

Owing to our condition that $V\left(c_{0}, c_{1}, \cdots, c_{n}\right) \cap U=\varnothing$, we know that at least one of the coefficients $c_{0}\left(\xi_{*}\right), \cdots, c_{n}\left(\xi_{*}\right)$ is nonzerd 4 . Thus we now have a solution $T_{K}$ to an ODE with constant coefficients. But then $T_{K}$ is a classical smooth solution expressible as a linear combination of analytic functions of the type $t^{k} e^{\lambda t}$ for some nonnegative integers $k$ and some complex numbers $\lambda$. The zero past condition $\left.T_{K}\right|_{(-T, 0)}=\mathbf{0}$, furthermore implies that this analytic function must in fact be identically zero, that is $T_{K}=\mathbf{0}$ in $(-T, T)$, a contradiction. Hence our assumption that $w$ is nonzero can't be true. Consequently, $w=\mathbf{0}$. This completes the proof of the lemma when $d=1$.

Step 2. Suppose now that $d>1$, and that the statement of the lemma holds for all spatial dimensions strictly less than $d$. We wish to prove the induction step that then the result holds for $d$-many spatial dimensions too. Let $w$ be a solution to

$$
c_{0}(\boldsymbol{\xi})+c_{1}(\boldsymbol{\xi}) \frac{\partial}{\partial t} w+\cdots+c_{n}(\boldsymbol{\xi}) \frac{\partial^{n}}{\partial t^{n}} w=\mathbf{0},
$$

with zero past. Suppose that $w$ is nonzero. Holmgren's uniqueness theorem (Lemma 2.2) implies that

$$
\operatorname{supp}(w) \subset\left\{(\boldsymbol{\xi}, t) \in U \times \mathbb{R}: c_{n}(\boldsymbol{\xi})=0, t \geqslant 0\right\} .
$$

If $c_{n}$ is constant (which must necessarily be nonzero, since $c_{n}$ is nonzero), then $w=\mathbf{0}$, a contradiction, and so we are done.

Suppose that $c_{n}$ is not a constant. Then we can decompose $V\left(c_{n}\right)$ as

$$
V\left(c_{n}\right):=M_{d-1} \cup \cdots \cup M_{0},
$$

where $M_{k}$ is the union of $k$-dimensional real analytic varieties, each possessing an analytic parametrisation, as in Subsection 2.3.

Let $\boldsymbol{\xi}_{*} \in M_{d^{\prime}}$, where $0<d^{\prime} \leqslant d-1$. Then there exists an open neighbourhood $\Omega$ of $\boldsymbol{\xi}_{*}$, an open neighbourhood $W$ of $\mathbf{0} \in \mathbb{R}^{d}$, and a homeomorphism

$$
\mathbb{R}^{d^{\prime}} \times \mathbb{R}^{d-d^{\prime}} \supset W \ni(\boldsymbol{\tau}, \boldsymbol{\sigma}) \mapsto \boldsymbol{\xi}(\boldsymbol{\tau}, \boldsymbol{\sigma}): W \rightarrow \Omega,
$$

\footnotetext{
${ }^{4}$ We know that $c_{n}\left(\xi_{*}\right)=0$ since $\xi_{*}$ was one of the roots of $c_{n}$.
} 


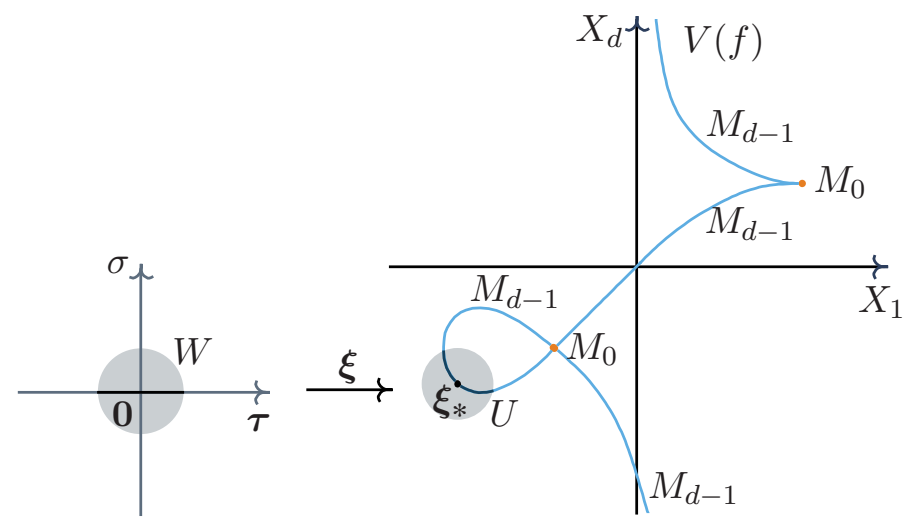

such that $\boldsymbol{\tau} \mapsto \boldsymbol{\xi}(\boldsymbol{\tau}, \mathbf{0}) \in \mathrm{C}^{\omega}(\widetilde{\Omega})$, where

$$
\widetilde{\Omega}=\left\{\boldsymbol{\tau} \in \mathbb{R}^{d^{\prime}} \text { such that }(\boldsymbol{\tau}, \mathbf{0}) \in W\right\},
$$

and $M_{d-1} \cap \Omega=\{\boldsymbol{\xi}(\boldsymbol{\tau}, \mathbf{0}):(\boldsymbol{\tau}, \mathbf{0}) \in W\}$. Suppose that $w$ is nonzero in $\Omega \times \mathbb{R}$. Then there is a large enough $T>0$ such that $w$ is nonzero on $\Omega \times(-T, T)$. By the Schwartz structure theorem from Subsection 2.2, we can decompose $w$ locally in a neighbourhood $\Omega \times(-T, T)$ of $\left(\boldsymbol{\xi}_{*}, 0\right) \in \mathbb{R}^{d+1}$ as

$$
w=\sum_{|\mathbf{k}| \leqslant K} \partial_{\boldsymbol{\sigma}}^{\mathbf{k}} T_{\mathbf{k}},
$$

for some distributions $T_{\mathbf{k}}$ on $\left(M_{d^{\prime}} \cap \Omega\right) \times(-T, T)$, such that not all $T_{\mathbf{k}}=\mathbf{0}$ when $|\mathbf{k}|=K$, Moreover, as $\left.w\right|_{t<0}=\mathbf{0}$, it follows that $\left.T_{\mathbf{k}}\right|_{t<0}=\mathbf{0}$ for each $\mathbf{k}$. Now for a $d-d^{\prime}$ tuple $\mathbf{k}=\left(k_{d^{\prime}+1}, \cdots, k_{d}\right)$ of nonnegative integers, with $|\mathbf{k}|=K$, let $\psi_{\mathbf{k}}$ be the smooth function

$$
\psi_{\mathbf{k}}:=\sigma_{1}^{k_{d^{\prime}}+1} \cdots \sigma_{d-d^{\prime}}^{k_{d}} .
$$

Then

$$
\left.\partial_{\boldsymbol{\sigma}}^{\mathbf{k}^{\prime}} \psi_{\mathbf{k}}\right|_{\boldsymbol{\sigma}=\mathbf{0}}=k_{d^{\prime}+1} ! \cdots k_{d} ! \cdot \delta_{k_{d^{\prime}+1}, k_{d^{\prime}+1}^{\prime}} \cdots \delta_{k_{d}, k_{d}^{\prime}}
$$

where $\delta_{\ell, \ell^{\prime}}$ denotes the Kronecker delta, defined to be 1 if $\ell=\ell^{\prime}$, and 0 otherwise. Using

$$
c_{0}(\boldsymbol{\xi})+c_{1}(\boldsymbol{\xi}) \frac{\partial}{\partial t} w+\cdots+c_{n}(\boldsymbol{\xi}) \frac{\partial^{n}}{\partial t^{n}} w=\mathbf{0}
$$

it follows that for all $\varphi \in \mathcal{D}(\widetilde{\Omega} \times(-T, T))$, where $\widetilde{\Omega}$ is as defined in ([6), we have

$$
\begin{aligned}
0 & =\left\langle\sum_{\ell=0}^{n} \sum_{\left|\mathbf{k}^{\prime}\right| \leqslant K} c_{\ell}(\boldsymbol{\xi}(\boldsymbol{\tau}, \boldsymbol{\sigma}))\left(\frac{\partial}{\partial t}\right)^{\ell} \partial_{\boldsymbol{\sigma}}^{\mathbf{k}^{\prime}} T_{\mathbf{k}^{\prime}}, \psi_{\mathbf{k}} \otimes \varphi\right\rangle \\
& =\sum_{\ell=0}^{n}\left\langle(-1)^{K} k_{d^{\prime}+1} ! \cdots k_{d} ! c_{\ell}(\boldsymbol{\xi}(\boldsymbol{\tau}, \mathbf{0}))\left(\frac{\partial}{\partial t}\right)^{\ell} T_{\mathbf{k}}, \varphi\right\rangle .
\end{aligned}
$$


But as $\boldsymbol{\tau} \mapsto \boldsymbol{\xi}(\boldsymbol{\tau}, \mathbf{0}) \in \mathrm{C}^{\omega}(\widetilde{\Omega})$, and each $c_{\ell} \in \mathrm{C}^{\omega}(U)$, it follows that their (well-defined) composition $\boldsymbol{\tau} \mapsto c_{\ell}(\boldsymbol{\xi}(\boldsymbol{\tau}, \mathbf{0}))$ is real analytic too. Thanks to the assumption that $V\left(c_{0}, c_{1}, \cdots, c_{n}\right) \cap U=\varnothing$, we also obtain in particular that with $\widetilde{c}_{\ell}(\boldsymbol{\tau}):=c_{\ell}(\boldsymbol{\xi}(\boldsymbol{\tau}, \mathbf{0})), \ell=0,1, \cdots, n$, and with

$$
p_{0}:=\sum_{\ell=0}^{n} \widetilde{c}_{\ell}(\boldsymbol{\xi}(\boldsymbol{\tau}, 0)) T^{\ell} \in \mathrm{C}^{\omega}(\widetilde{\Omega})[T],
$$

we have $V\left(\widetilde{c}_{0}, \widetilde{c}_{1}, \cdots, \widetilde{c}_{n}\right) \cap \mathbb{R}^{d^{\prime}}=\varnothing$, and $D_{p_{0}} T_{\mathbf{k}}=\mathbf{0}$. We also recall from the above that $\left.T_{\mathbf{k}}\right|_{t<0}=\mathbf{0}$. By the induction hypothesis, we conclude that $T_{\mathbf{k}}=\mathbf{0}$. Repeating this argument for each $\mathbf{k}$ satisfying $|\mathbf{k}|=K$, gives $T_{\mathbf{k}}=\mathbf{0}$ whenever $|\mathbf{k}|=K$. But this is a contradiction. This means that $M_{d^{\prime}} \cap \operatorname{supp}(w)=\varnothing$. As $d^{\prime}$ such that $0<d^{\prime} \leqslant d-1$ was arbitrary, we conclude that $\operatorname{supp}(w) \subset M_{0}$. But now we repeat the same argument above from Step 1, when $w$ was supported on isolated lines, to conclude that $\operatorname{supp}(w)=\varnothing$, that is, $w=\mathbf{0}$. This completes the induction step, and the proof of the lemma.

\section{Proof of Sufficiency}

In Theorem 1.9, we had seen that the condition $V\left(C_{\mathbf{X}}(p)\right) \cap i \mathbb{R}^{d}=\varnothing$ is necessary for the triviality of the null solution space $N_{\mathcal{L}\left(\mathcal{D}(\mathbb{R}), \mathcal{S}^{\prime}\left(\mathbb{R}^{d}\right)\right)}(p)=\{\mathbf{0}\}$. We now show that this condition is also sufficient.

\section{Theorem 4.1.}

Let $p=a_{0}(\mathbf{X})+a_{1}(\mathbf{X}) T+\cdots+a_{n}(\mathbf{X}) T^{n} \in \mathbb{C}[\mathbf{X}][T]$ such that $a_{n} \neq \mathbf{0} \in \mathbb{C}[\mathbf{X}]$. If $V\left(C_{\mathbf{X}}(p)\right) \cap i \mathbb{R}^{d}=\varnothing$, then $N_{\mathcal{L}\left(\mathcal{D}(\mathbb{R}), \mathcal{S}^{\prime}\left(\mathbb{R}^{d}\right)\right)}(p)=\{\mathbf{0}\}$.

Proof. Suppose that $V\left(C_{\mathbf{X}}(p)\right) \cap i \mathbb{R}^{d}=\varnothing$. Let $u \in \mathcal{L}\left(\mathcal{D}(\mathbb{R}), \mathcal{S}^{\prime}\left(\mathbb{R}^{d}\right)\right)$ be such that $\left.u\right|_{t<0}=\mathbf{0}, D_{p} u=\mathbf{0}$ and such that $u \neq \mathbf{0}$. Upon taking Fourier transformation on both sides of the equation $D_{p} u=\mathbf{0}$ with respect to the spatial variables, we obtain

$$
a_{0}(i \boldsymbol{\xi}) \widehat{u}+a_{1}(i \boldsymbol{\xi}) \frac{\partial}{\partial t} \widehat{u}+\cdots+a_{n}(i \boldsymbol{\xi})\left(\frac{\partial}{\partial t}\right)^{n} \widehat{u}=\mathbf{0} .
$$

By Lemma 3.1, this implies $\widehat{u}=\mathbf{0}$. Taking the inverse Fourier transform yields $u=\mathbf{0}$, completing the proof.

Example 4.2 (Diffusion equation revisited). Consider the diffusion equation

$$
\left(\frac{\partial}{\partial t}-\Delta\right) u=\mathbf{0}
$$

that is, $D_{p} u=\mathbf{0}$, where $p(\mathbf{X}, T)=T-\left(X_{1}^{2}+\cdots+X_{d}^{2}\right)$.

The constant polynomial $a_{1}=\mathbf{1}$ is nonzero, and so $V\left(C_{\mathbf{X}}(p)\right) \cap i \mathbb{R}^{d}=\varnothing$. Theorem 4.1 implies that $N_{\mathcal{L}\left(\mathcal{D}(\mathbb{R}), \mathcal{S}^{\prime}\left(\mathbb{R}^{d}\right)\right)}(p)=\{\mathbf{0}\}$, in conformity with our physical intuition. 
Modern physics rejects the diffusion equation as an accurate model of physical reality since it is not 'Lorentz invariant', admitting infinite propagation speeds. This can already be seen in the case of classical solutions to the initial value problem to the diffusion equation, where the solution is given by a (spatial) convolution of the initial data $f$ with the Gaussian kernel, and so for arbitrarily small time instants $t>0$ and at $\mathbf{x}=\mathbf{0}$, even arbitrarily far away initial data has an influence, which violates the special relativistic tenet that nothing travels faster than the speed of light. With this in mind, we choose to illustrate our main theorem also with the Lorentz invariant Klein-Gordon equation.

Example 4.3 (Klein-Gordon equation on Minkowski space). For $m \in \mathbb{R}$, consider the equation

$$
\left(\frac{\partial^{2}}{\partial t^{2}}-\Delta+m^{2}\right) u=\mathbf{0}
$$

that is, $D_{p} u=\mathbf{0}$, where $p(\mathbf{X}, T)=T^{2}-\left(X_{1}^{2}+\cdots+X_{d}^{2}\right)+m^{2}$.

The constant polynomial $a_{2}=\mathbf{1}$ is nonzero, and so $V\left(C_{\mathbf{X}}(p)\right) \cap i \mathbb{R}^{d}=\varnothing$. Theorem 4.1 implies that $N_{\mathcal{L}\left(\mathcal{D}(\mathbb{R}), \mathcal{S}^{\prime}\left(\mathbb{R}^{d}\right)\right)}(p)=\{\mathbf{0}\}$.

We remark that Proposition 1.4 also gives a sensible result in this case, since

$\operatorname{deg}(p)=\operatorname{deg}\left(T^{2}-\left(X_{1}^{2}+\cdots+X_{d}^{2}\right)+m^{2}\right)=2=\operatorname{deg}\left(T^{2}+m^{2}\right)=\operatorname{deg}(p(\mathbf{0}, T))$, and so $N_{\mathcal{D}^{\prime}\left(\mathbb{R}^{d+1}\right)}(p)=\{\mathbf{0}\}$ and $N_{\mathrm{C}^{\infty}\left(\mathbb{R}^{d+1}\right)}(p)=\{\mathbf{0}\}$.

If $\eta_{\mu \nu}(\mu, \nu=0,1,2,3)$ are the Minkowski metric tensor components in the Cartesian/inertial coordinates, then the only Lorentz-invariant scalar linear constant coefficient differential operator one can build has the form

$$
\sum_{n=0}^{N} c_{n}\left(\eta^{\mu \nu} \partial_{\mu} \partial_{\nu}\right)^{n}
$$

where $\left[\eta^{\mu \nu}\right]$ denotes the inverse of the metric matrix $\left[\eta_{\mu \nu}\right]$, and $c_{k} \in \mathbb{C}$. This corresponds to the polynomial

$$
p=\sum_{n=0}^{N} c_{n}\left(T^{2}-\left(X_{1}^{2}+\cdots+X_{d}^{2}\right)\right)^{n},
$$

and so $\operatorname{deg}(p)=\operatorname{deg}(p(\mathbf{0}, T))$ is always satisfied for such Lorentz invariant partial differential operators. Thus Hörmander's Proposition 1.4 is physically sound from the spacetime perspective of special relativity.

Example 4.4. Consider the equation

$$
\frac{\partial^{d+1}}{\partial t \partial x_{1} \cdots \partial x_{d}} u=\mathbf{0}
$$

that is, $D_{p} u=\mathbf{0}$, where $p(\mathbf{X}, T)=X_{1} \cdots X_{d} T$. Then

$$
V\left(C_{\mathbf{X}}(p)\right)=\bigcup_{k=1}^{d} \operatorname{span}_{\mathbb{C}}\left(\mathbf{e}_{k}\right),
$$


where $\mathbf{e}_{k}$ is the standard basis vector in $\mathbb{C}^{d}$ with 1 in the $k$ th entry, and all other entries zeroes. Thus $V\left(C_{\mathbf{X}}(p)\right) \cap i \mathbb{R}^{d} \neq \varnothing$, so that

$$
N_{\mathcal{L}\left(\mathcal{D}(\mathbb{R}), \mathcal{S}^{\prime}\left(\mathbb{R}^{d}\right)\right)}(p) \neq\{\mathbf{0}\} .
$$

This is expected, and we can easily construct nontrivial null solutions as in the proof of Theorem 1.9. In fact, with 1 denoting the constant function on $\mathbb{R}^{d}$ taking value 1 everywhere, we can take $u:=\mathbf{1} \otimes \Theta(t)$, where $\Theta$ is as in (2). Then $\mathbf{0} \neq u \in N_{\mathcal{L}\left(\mathcal{D}(\mathbb{R}), \mathcal{S}^{\prime}\left(\mathbb{R}^{d}\right)\right)}(p)$ since it has zero past and satisfies $D_{p} u=\mathbf{0}$.

\section{Spatial profile in Besov spaces}

We mention that besides the space $\mathcal{L}\left(\mathcal{D}(\mathbb{R}), \mathcal{S}^{\prime}\left(\mathbb{R}^{d}\right)\right.$ ), one may consider also other natural solution spaces with some growth restriction in the spatial directions. As an example, we consider $\mathcal{L}\left(\mathcal{D}(\mathbb{R}), B_{p, k}\left(\mathbb{R}^{d}\right)\right)$, where $B_{p, k}\left(\mathbb{R}^{d}\right)$ is a subspace of $\mathcal{S}^{\prime}\left(\mathbb{R}^{d}\right)$ defined below.

We follow [8, §10.1]. A positive function $k$ defined on $\mathbb{R}^{d}$ will be called a temperate weight function if there exist positive constants $C$ and $N$ such that

$$
k(\boldsymbol{\xi}+\boldsymbol{\eta}) \leqslant\left(1+C\|\boldsymbol{\xi}\|_{2}\right)^{N} k(\boldsymbol{\eta}), \quad \boldsymbol{\xi}, \boldsymbol{\eta} \in \mathbb{R}^{d},
$$

where $\|\cdot\|_{2}$ denotes the Euclidean norm on $\mathbb{R}^{d}$. The set of all such functions will be denoted by $\mathcal{K}$. If $k \in \mathcal{K}$ and $1 \leqslant p \leqslant \infty$, then the Besov space $B_{p, k}$ is the set of all distributions $u \in \mathcal{S}^{\prime}\left(\mathbb{R}^{d}\right)$ such that the Fourier transform $\widehat{u}$ of $u$ is a function and

$$
\|u\|_{p, k}=\left(\int_{\mathbb{R}^{d}}|k(\boldsymbol{\xi}) \widehat{u}(\boldsymbol{\xi})|^{p} \mathrm{~d}^{d} \boldsymbol{\xi}\right)^{1 / p}<\infty .
$$

When $p=\infty$, we take $\|u\|_{p, k}$ as ess.sup $|k(\cdot) \widehat{u}(\cdot)|$. Then $B_{p, k}$ is a Banach space with the above norm. The usual scale of Sobolev space $H_{s}\left(\mathbb{R}^{d}\right)$ parameterised by real numbers $s$ corresponds to the class

$$
\mathcal{K}_{\text {Sob }}:=\left\{k_{s}: s \in \mathbb{R}\right\} \subset \mathcal{K},
$$

where

$$
k_{s}(\boldsymbol{\xi}):=\left(1+\|\boldsymbol{\xi}\|^{2}\right)^{s / 2}
$$

We can think of the space $\mathcal{L}\left(\mathcal{D}(\mathbb{R}), B_{p, k}\left(\mathbb{R}^{d}\right)\right)$ as a subspace of $\mathcal{D}^{\prime}\left(\mathbb{R}^{d+1}\right)$ : if $u \in \mathcal{L}\left(\mathcal{D}(\mathbb{R}), B_{p, k}\left(\mathbb{R}^{d}\right)\right)$, then we define the distribution $U \in \mathcal{D}^{\prime}\left(\mathbb{R}^{d+1}\right)$ by

$$
\langle U, \psi \otimes \varphi\rangle=\int_{\mathbb{R}^{d}}(\langle u, \varphi\rangle)(\boldsymbol{\xi}) \psi(\boldsymbol{\xi}) \mathrm{d}^{d} \boldsymbol{\xi}
$$

for $\varphi \in \mathcal{D}(\mathbb{R}), \psi \in \mathcal{D}\left(\mathbb{R}^{d}\right)$.

We prove the following result. Despite again using the Fourier transform as the main tool, akin to the proof of Lemma 3.1. the proof is markedly simpler, thanks to the possibility of 'evaluation' at $\boldsymbol{\xi}$ (since for every 'time' test function $\varphi \in \mathcal{D}(\mathbb{R})$, we have that $\widehat{u}(\varphi)$ is a function of the variable $\left.\xi \in \mathbb{R}^{d}\right)$. 
Theorem 5.1.

Let $p \in \mathbb{C}[\mathbf{X}, T]$. Then $N_{\mathcal{L}\left(\mathcal{D}(\mathbb{R}), B_{p, k}\left(\mathbb{R}^{d}\right)\right)}(p)=\{\mathbf{0}\}$ if and only if $p \neq \mathbf{0}$.

Proof. 'Only if' part: Suppose that $p=\mathbf{0}$. Then we just take any nonzero $\psi \in B_{p, k}$. For example, any nonzero $\psi \in \mathcal{D}\left(\mathbb{R}^{d}\right)$ will do. Moreover, let $\Theta$ be the nonzero function in $\mathrm{C}^{\infty}(\mathbb{R})$ which has a zero past given in (2). Define $u$ by $u(x, t):=\psi(x) \Theta(t)$ for $x \in \mathbb{R}^{d}$ and $t \in \mathbb{R}$. Then we have that $u \in \mathcal{L}\left(\mathcal{D}(\mathbb{R}), B_{p, k}\left(\mathbb{R}^{d}\right)\right),\left.u\right|_{t<0}=\mathbf{0}$ and $D_{p} u=\mathbf{0}$. But $u \neq \mathbf{0}$, and so $N_{\mathcal{L}\left(\mathcal{D}(\mathbb{R}), B_{p, k}\left(\mathbb{R}^{d}\right)\right)}(p) \neq\{\mathbf{0}\}$.

'If' part: Suppose that $p \neq \mathbf{0}$. Let $u \in \mathcal{L}\left(\mathcal{D}(\mathbb{R}), B_{p, k}\left(\mathbb{R}^{d}\right)\right)$ be such that $\left.u\right|_{t<0}=\mathbf{0}$ and $D_{p} u=\mathbf{0}$. Let $p=a_{0}+a_{1} T+\cdots+a_{n} T^{n} \in \mathbb{C}[\mathbf{X}][T]$, where $a_{0}, a_{1}, \cdots, a_{n} \in \mathbb{C}[\mathbf{X}]$ and $a_{n} \neq \mathbf{0}$ in $\mathbb{C}[\mathbf{X}]$. Upon taking Fourier transformation on both sides of the equation $D_{p} u=\mathbf{0}$ with respect to the spatial variables, we obtain

$$
a_{0}(i \boldsymbol{\xi}) \widehat{u}+a_{1}(i \boldsymbol{\xi}) \frac{\partial}{\partial t} \widehat{u}+\cdots+a_{n}(i \boldsymbol{\xi})\left(\frac{\partial}{\partial t}\right)^{n} \widehat{u}=\mathbf{0} .
$$

But if we fix a $\boldsymbol{\xi} \in \mathbb{R}^{d}$ such that $a_{n}(i \boldsymbol{\xi}) \neq 0$, then it follows that $(\widehat{u}(\varphi))(\boldsymbol{\xi})=0$ for all $\varphi \in \mathcal{D}(\mathbb{R})$. Since the Lebesgue measure of the set of zeros of the polynomial function $a_{n}(i \boldsymbol{\xi})$ is zero, it follows that for each $\varphi \in \mathcal{D}(\mathbb{R})$, the function $\mathbb{R}^{d} \ni \boldsymbol{\xi} \mapsto(\widehat{u}(\varphi))(\boldsymbol{\xi})$ almost everywhere, and so $\widehat{u}(\varphi)=\mathbf{0}$. But then $\widehat{u}=\mathbf{0}$ too, and so $u=\mathbf{0}$. This completes the proof.

And immediate consequence is the following, since the $H_{s}\left(\mathbb{R}^{d}\right)$ are all special instances of the Besov spaces $B_{p, q}\left(\mathbb{R}^{d}\right)$ [8, Example 10.1.2, p.5], and also $\mathcal{S}\left(\mathbb{R}^{d}\right) \subset B_{p, k}\left(\mathbb{R}^{d}\right)$ [8, Thm. 10.1.7, p.7].

\section{Corollary 5.2.}

Let $p \in \mathbb{C}[\mathbf{X}, T]$ and $S=\mathcal{L}\left(\mathcal{D}(\mathbb{R}), H_{s}\left(\mathbb{R}^{d}\right)\right)$ or $S=\mathcal{L}\left(\mathcal{D}(\mathbb{R}), \mathcal{S}\left(\mathbb{R}^{d}\right)\right)$.

Then $N_{S}(p)=\{\mathbf{0}\}$ if and only if $p \neq \mathbf{0}$.

By the Payley-Wiener-Schwartz theorem [12, Prop. 29.1, p. 307], we know that the Fourier transform of elements of $\mathcal{E}^{\prime}\left(\mathbb{R}^{d}\right)$ can be extended to entire functions on $\mathbb{C}^{d}$. Thus the same proof, mutatis mutandis, as that of Theorem 5.1 gives the following.

\section{Theorem 5.3.}

Let $p \in \mathbb{C}[\mathbf{X}, T]$. Then $N_{\mathcal{L}\left(\mathcal{D}(\mathbb{R}) \mathcal{E}^{\prime}\left(\mathbb{R}^{d}\right)\right)}(p)=\{\mathbf{0}\}$ if and only if $p \neq \mathbf{0}$.

\section{Spatially PERIOdiC Distributions}

In this final section, we consider the space $\mathcal{D}_{\mathbb{A}}^{\prime}\left(\mathbb{R}^{d+1}\right)$, which is, roughly speaking, the set of all distributions on $\mathbb{R}^{d+1}$ that are periodic in the spatial directions with a discrete set $\mathbb{A}$ of periods. The precise definition of $\mathcal{D}_{\AA}^{\prime}\left(\mathbb{R}^{d+1}\right)$ is given below.

For $\mathbf{a} \in \mathbb{R}^{d}$, the translation operation $\mathbf{S}_{\mathbf{a}}$ on distributions in $\mathcal{D}^{\prime}\left(\mathbb{R}^{d}\right)$ is defined by

$$
\left\langle\mathbf{S}_{\mathbf{a}}(T), \varphi\right\rangle=\langle T, \varphi(\cdot+\mathbf{a})\rangle \text { for all } \varphi \in \mathcal{D}\left(\mathbb{R}^{d}\right) .
$$


A distribution $T \in \mathcal{D}^{\prime}\left(\mathbb{R}^{d}\right)$ is said to be periodic with a period $\mathbf{a} \in \mathbb{R}^{d}$ if $T=\mathbf{S}_{\mathbf{a}}(T)$.

Let $\mathbb{A}:=\left\{\mathbf{a}_{\mathbf{1}}, \cdots, \mathbf{a}_{\mathbf{d}}\right\}$ be a linearly independent set vectors in $\mathbb{R}^{d}$. We define $\mathcal{D}_{\mathbb{A}}^{\prime}\left(\mathbb{R}^{d}\right)$ to be the set of all distributions $T$ that satisfy

$$
\mathbf{S}_{\mathbf{a}_{\mathbf{k}}}(T)=T, \quad k=1, \cdots, d .
$$

From [2, §34], $T$ is a tempered distribution, and from the above it follows by taking Fourier transforms that $\left(1-e^{i \mathbf{a}_{\mathbf{k}} \cdot \boldsymbol{\xi}}\right) \widehat{T}=0$ for $k=1, \cdots, d$. It can be seen that

$$
\widehat{T}=\sum_{\mathbf{v} \in A^{-1} 2 \pi \mathbb{Z}^{d}} \alpha_{\mathbf{v}}(T) \delta_{\mathbf{v}}
$$

for some scalars $\alpha_{\mathbf{v}} \in \mathbb{C}$, and where $A$ is the matrix with its rows equal to the transposes of the column vectors $\mathbf{a}_{\mathbf{1}}, \cdots, \mathbf{a}_{\mathbf{d}}$ :

$$
A:=\left[\begin{array}{c}
\mathbf{a}_{\mathbf{1}}^{\top} \\
\vdots \\
\mathbf{a}_{\mathbf{d}}^{\top}
\end{array}\right] .
$$

By the Schwartz Kernel Theorem [7, p. 128, Theorem 5.2.1], we know that $\mathcal{D}^{\prime}\left(\mathbb{R}^{d+1}\right)$ is isomorphic as a topological space to $\mathcal{L}\left(\mathcal{D}(\mathbb{R}), \mathcal{D}^{\prime}\left(\mathbb{R}^{d}\right)\right)$, the space of all continuous linear maps from $\mathcal{D}(\mathbb{R})$ to $\mathcal{D}^{\prime}\left(\mathbb{R}^{d}\right)$, thought of as vectorvalued distributions. In this section, we indicate this isomorphism by putting an arrow on top of elements of $\mathcal{D}^{\prime}\left(\mathbb{R}^{d+1}\right)$. Thus for $u \in \mathcal{D}^{\prime}\left(\mathbb{R}^{d+1}\right)$, we set $\vec{u} \in \mathcal{L}\left(\mathcal{D}(\mathbb{R}), \mathcal{D}^{\prime}\left(\mathbb{R}^{d}\right)\right)$ to be the vector valued distribution defined by

$$
\langle\vec{u}(\varphi), \psi\rangle=\langle u, \psi \otimes \varphi\rangle
$$

for $\varphi \in \mathcal{D}(\mathbb{R})$ and $\psi \in \mathcal{D}\left(\mathbb{R}^{d}\right)$. We define

$$
\mathcal{D}_{\mathbb{A}}^{\prime}\left(\mathbb{R}^{d+1}\right)=\left\{u \in \mathcal{D}^{\prime}\left(\mathbb{R}^{d+1}\right): \text { for all } \varphi \in \mathcal{D}(\mathbb{R}), \vec{u}(\varphi) \in \mathcal{D}_{\mathbb{A}}^{\prime}\left(\mathbb{R}^{d}\right)\right\} .
$$

Then for $u \in \mathcal{D}_{\mathbb{A}}^{\prime}\left(\mathbb{R}^{d+1}\right)$,

$$
\frac{\partial}{\partial x_{k}} u \in \mathcal{D}_{\mathbb{A}}^{\prime}\left(\mathbb{R}^{d+1}\right) \text { for } k=1, \cdots, d, \text { and } \frac{\partial}{\partial t} u \in \mathcal{D}_{\mathbb{A}}^{\prime}\left(\mathbb{R}^{d+1}\right) .
$$

Also, for $u \in \mathcal{D}_{\mathbb{A}}^{\prime}\left(\mathbb{R}^{d+1}\right)$, we define $\widehat{u} \in \mathcal{D}^{\prime}\left(\mathbb{R}^{d+1}\right)$ by

$$
\langle\widehat{u}, \psi \otimes \varphi\rangle=\langle\vec{u}(\varphi), \widehat{\psi}\rangle,
$$

for $\varphi \in \mathcal{D}(\mathbb{R})$ and $\psi \in \mathcal{D}\left(\mathbb{R}^{d}\right)$.

We have the following characterisation for the space of null solutions to be trivial.

\section{Theorem 6.1.}

Suppose that $\mathbb{A}=\left\{\mathbf{a}_{\mathbf{1}}, \cdots, \mathbf{a}_{\mathbf{d}}\right\}$ is a linearly independent set of vectors in $\mathbb{R}^{d}$. Let $S=\mathcal{D}_{\mathbb{A}}^{\prime}\left(\mathbb{R}^{d+1}\right)$ and $p \in \mathbb{C}[\mathbf{X}, T]$.

Then $N_{S}(p)=\{\mathbf{0}\}$ if and only if for all $\mathbf{v} \in A^{-1} 2 \pi \mathbb{Z}^{d}$, there exists a $t \in \mathbb{C}$ such that $p(i \mathbf{v}, t) \neq 0$. 
Proof.

'Only if' part: Let $\mathbf{v}_{\mathbf{0}} \in A^{-1} 2 \pi \mathbb{Z}^{d}$ be such that for all $t \in \mathbb{C}, p\left(i \mathbf{v}_{\mathbf{0}}, t\right)=\mathbf{0}$. Then $p\left(i \mathbf{v}_{\mathbf{0}}, T\right)$ is the zero polynomial in $\mathbb{C}[T]$. Let $\Theta \in C^{\infty}(\mathbb{R})$ be any nonzero smooth function such that $\left.\Theta\right|_{t<0}=\mathbf{0}$. Define $u:=e^{i \mathbf{v}_{\mathbf{0}} \cdot \mathbf{x}} \otimes \Theta$. Here $\mathbf{v}_{\mathbf{0}} \cdot \mathbf{x}$ is the usual Euclidean inner product of $\mathbf{v}_{\mathbf{0}}$ and $\mathbf{x}$ in the real vector space $\mathbb{R}^{d}$. Then we have that $u \in \mathcal{D}_{\mathbb{A}}^{\prime}\left(\mathbb{R}^{d+1}\right)$, since

$$
\mathbf{S}_{\mathbf{a}_{\mathbf{k}}} u=e^{i \mathbf{v}_{\mathbf{0}} \cdot\left(\mathbf{x}+\mathbf{a}_{\mathbf{k}}\right)} \otimes \Theta=e^{i \mathbf{v}_{\mathbf{0}} \cdot \mathbf{a}_{\mathbf{k}}} e^{i \mathbf{v}_{\mathbf{0}} \cdot \mathbf{x}} \otimes \Theta=1 \cdot e^{i \mathbf{v}_{\mathbf{0}} \cdot \mathbf{x}} \otimes \Theta=u .
$$

Moreover, $u$ has zero past, that is, $\left.u\right|_{t<0}=\mathbf{0}$ because $\left.\Theta\right|_{t<0}=\mathbf{0}$. Also, the trajectory $u \in N_{\mathcal{D}_{\mathbb{A}}^{\prime}\left(\mathbb{R}^{d+1}\right)}(p) \backslash\{\mathbf{0}\}$ because $\Theta \neq \mathbf{0}$ and

$$
p\left(\frac{\partial}{\partial x_{1}}, \cdots, \frac{\partial}{\partial x_{d}}, \frac{\partial}{\partial t}\right) u=e^{i \mathbf{v}_{\mathbf{0}} \cdot \mathbf{x}} p\left(i \mathbf{v}_{\mathbf{0}}, \frac{d}{d t}\right) \Theta=e^{i \mathbf{v}_{\mathbf{0}} \cdot \mathbf{x}} \cdot \mathbf{0}=\mathbf{0} .
$$

This completes the proof of the 'only if' part.

('If' part:) On the other hand, now suppose that for each $\mathbf{v} \in A^{-1} 2 \pi \mathbb{Z}^{d}$, there exists a $t \in \mathbb{C}$ such that $p(i \mathbf{v}, t) \neq 0$. Then the polynomial $p(i \mathbf{v}, T)$ is not the zero polynomial in $\mathbb{C}[T]$. So $N_{\mathcal{D}^{\prime}(\mathbb{R})}(p(i \mathbf{v}, T))=\{\mathbf{0}\}$. Thus for each $\mathbf{v} \in A^{-1} 2 \pi \mathbb{Z}^{d}$, whenever a distribution $T \in \mathcal{D}^{\prime}(\mathbb{R})$ is such that $\left.T\right|_{t<0}=\mathbf{0}$ and satisfies

$$
p\left(i \mathbf{v}, \frac{d}{d t}\right) T=\mathbf{0}
$$

there holds that $T=\mathbf{0}$. Now suppose that $u \in \mathcal{D}_{\mathbb{A}}^{\prime}\left(\mathbb{R}^{d+1}\right)$ satisfies $\left.u\right|_{t<0}=\mathbf{0}$ and

$$
p\left(\frac{\partial}{\partial x_{1}}, \cdots, \frac{\partial}{\partial x_{d}}, \frac{\partial}{\partial t}\right) u=\mathbf{0} .
$$

Upon taking Fourier transformation on both sides of the equation (9) with respect to the spatial variables, we obtain

$$
p\left(i \boldsymbol{\xi}, \frac{\partial}{\partial t}\right) \widehat{u}=\mathbf{0}
$$

For each fixed $\varphi \in \mathcal{D}(\mathbb{R}), \vec{u}(\varphi) \in \mathcal{D}_{\mathbb{A}}^{\prime}\left(\mathbb{R}^{d}\right)$, and so it follows that

$$
\widehat{\vec{u}(\varphi)}=\sum_{\mathbf{v} \in A^{-1} 2 \pi \mathbb{Z}^{d}} \delta_{\mathbf{v}} \alpha_{\mathbf{v}}(\widehat{u}, \varphi)
$$

for appropriate coefficients $\alpha_{\mathbf{v}}(\widehat{u}, \varphi) \in \mathbb{C}$. In particular, we see that the support of $\widehat{u}$ is contained in $A^{-1} 2 \pi \mathbb{Z}^{d} \times[0,+\infty)$. Thus each of the half lines in $A^{-1} 2 \pi \mathbb{Z}^{d} \times[0,+\infty)$ carries a solution of the differential equation (10), and $\widehat{u}$ is a sum of these. We will show that each of these summands is zero. It can be seen from (11) that the map $\varphi \mapsto \alpha_{\mathbf{v}}(\widehat{u}, \varphi): \mathcal{D}(\mathbb{R}) \rightarrow \mathbb{C}$ defines a distribution $T^{(\mathbf{v})}$ in $\mathcal{D}^{\prime}(\mathbb{R})$. Moreover, the support of $T^{(\mathbf{v})}$ is contained in $[0,+\infty)$. From (11), we see that for a small enough neighbourhood $N$ of $\mathbf{v} \in A^{-1} 2 \pi \mathbb{Z}^{d}$ in $\mathbb{R}^{d}$, we have

$$
\delta_{\mathbf{v}} \otimes p\left(i \mathbf{v}, \frac{d}{d t}\right) T^{(\mathbf{v})}=\mathbf{0}
$$


in $N \times \mathbb{R}$. But as we had seen above, our algebraic hypothesis implies that the set $N_{\mathcal{D}^{\prime}(\mathbb{R})}(p(i \mathbf{v}, T))$ of null solutions is trivial, i.e. $N_{\mathcal{D}^{\prime}(\mathbb{R})}(p(i \mathbf{v}, T))=\{\mathbf{0}\}$, and so $T^{(\mathbf{v})}=\mathbf{0}$. As this happens with each $\mathbf{v} \in A^{-1} 2 \pi \mathbb{Z}^{d}$, we conclude that $\widehat{u}=\mathbf{0}$ and hence also $u=\mathbf{0}$. Consequently, $N_{\mathcal{D}_{\mathbb{A}}^{\prime}\left(\mathbb{R}^{d+1}\right)}(p)=\{\mathbf{0}\}$.

\section{Open QUestion: For Which $p$ is the Set of FUtURes Of NUll} SOLUTIONS DENSE IN THE SET OF FUTURES OF ALL SOLUTIONS?

It follows from [5] that the set of futures of smooth null solutions, namely

$$
\left\{\left.u\right|_{t>0}: u \in N_{\mathrm{C}^{\infty}\left(\mathbb{R}^{d+1}\right)}(p)\right\}
$$

is dense in the set of futures of all smooth solutions, namely

$$
\left\{\left.u\right|_{t>0}: u \in \mathrm{C}^{\infty}\left(\mathbb{R}^{d+1}\right) \text { and } D_{p} u=\mathbf{0}\right\}
$$

if each irreducible factor $p^{\prime}$ of $p$ satisfies $\operatorname{deg}\left(p^{\prime}\right) \neq \operatorname{deg}\left(p^{\prime}(\mathbf{0}, T)\right)$.

In our alternative solution spaces, one could ask a similar question, namely if it is possible to give a characterisation in terms of the polynomial $p$ so that the set of futures of null solutions is dense in the set of futures of all solutions. We leave this class of an open questions for future investigation.

\section{Acknowledgements:}

I am grateful to Sara Maad Sasane and Arne Meurman (Lund University) for useful discussions.

\section{REFERENCES}

[1] E. Bierstone and P. Milman. Semianalytic and subanalytic sets. Institut des Hautes Études Scientifiques, Publications Mathématiques, 67:5-42, 1988.

[2] W.F. Donoghue, Jr. Distributions and Fourier Transforms. Pure and Applied Mathematics 32, Academic Press, New York and London, 1969.

[3] G. Hellwig. Partial differential equations. An introduction. Second edition. B.G. Teubner, 1977.

[4] L. Hörmander. On the theory of general partial differential operators. Acta Mathematica, 94:161-248, 1955.

[5] L. Hörmander. Null solutions of partial differential equations. Archive for Rational Mechanics and Analysis, 255-261 no. 4, 1960.

[6] L. Hörmander. Linear Partial Differential Operators. Fourth printing. Springer, 1976.

[7] L. Hörmander. The Analysis of Linear Partial Differential Operators. I. Second edition. Springer, 1990.

[8] L. Hörmander. The analysis of linear partial differential operators. II. Reprint of the 1983 original. Springer, 2005.

[9] S. Krantz and H. Parks. A Primer of Real Analytic Functions. Second edition. Birkhäuser, 2002.

[10] S. Łojasiewicz. Introduction to Complex Analytic Geometry. Translated from the Polish by Maciej Klimek. Birkhäuser, 1991.

[11] L. Schwartz. Théorie des Distributions. Hermann, Paris, 1966.

[12] F. Trèves. Topological Vector Spaces, Distributions and Kernels. Dover, New York, 2006.

[13] H. Whitney. Elementary structure of real algebraic varieties. Annals of Mathematics, Second Series, 66:545-556, 1957. 
Mathematics Department, London School of Economics, Houghton Street, LONDON WC2A 2AE, U.K.

E-mail address: A.J.Sasane@lse.ac.uk 